\title{
Spectral analysis of the barium central star of the planetary nebula Hen 2-39
}

\author{
L. Löbling ${ }^{1,2}$, H. M. J. Boffin ${ }^{1}$, and D. Jones ${ }^{3,4}$ \\ ${ }^{1}$ European Southern Observatory, Karl-Schwarzschild-Str. 2, 85748 Garching bei München, Germany \\ ${ }^{2}$ Institute for Astronomy and Astrophysics, Kepler Center for Astro and Particle Physics, Eberhard Karls University, Sand 1, \\ 72076 Tübingen, Germany \\ e-mail: loebling@astro.uni-tuebingen.de \\ ${ }^{3}$ Instituto de Astrofísica de Canarias, 38205 La Laguna, Tenerife, Spain \\ ${ }^{4}$ Departamento de Astrofísica, Universidad de La Laguna, 38206 La Laguna, Tenerife, Spain
}

Received 19 October 2018 / Accepted 4 February 2019

\begin{abstract}
Context. Barium stars are peculiar red giants characterized by an overabundance of the elements synthesized in the slow neutroncapture nucleosynthesis (s-process elements) along with an enrichment in carbon. These stars are discovered in binaries with white dwarf companions. The more recently formed of these stars are still surrounded by a planetary nebula.

Aims. Precise abundance determinations of the various s-process elements, of further key elements that act as indicators for effectiveness of nucleosynthesis on the asymptotic giant branch and, especially, of the lightest, short-lived radionuclide technetium will establish constraints for the formation of s-process elements in asymptotic giant branch stars as well as mass transfer through, for example, stellar wind, Roche-lobe overflow, and common-envelope evolution.

Methods. We performed a detailed spectral analysis of the K-type subgiant central star of the planetary nebula Hen 2-39 based on high-resolution optical spectra obtained with the Ultraviolet and Visual Echelle Spectrograph at the Very Large Telescope using local thermodynamic equilibrium model atmospheres.

Results. We confirm the effective temperature of $T_{\text {eff }}=(4350 \pm 150) \mathrm{K}$ for the central star of the planetary nebula Hen $2-39$. It has a photospheric carbon enrichment of $[\mathrm{C} / \mathrm{H}]=0.36 \pm 0.08$ and a barium overabundance of $[\mathrm{Ba} / \mathrm{Fe}]=1.8 \pm 0.5$. We find a deficiency for most of the iron-group elements (calcium to iron) and establish an upper abundance limit for technetium $\left(\log \epsilon_{\mathrm{Tc}}<2.5\right)$.

Conclusions. The quality of the available optical spectra is not sufficient to measure abundances of all s-process elements accurately. Despite large uncertainties on the abundances as well as on the model yields, the derived abundances are most consistent with a progenitor mass in the range $1.75-3.00 M_{\odot}$ and a metallicity of $[\mathrm{Fe} / \mathrm{H}]=-0.3 \pm 1.0$. This result leads to the conclusion that the formation of such systems requires a relatively large mass transfer that is most easily obtained via wind-Roche lobe overflow.
\end{abstract}

Key words. planetary nebulae: individual: Hen 2-39 - stars: abundances - stars: evolution - stars: AGB and post-AGB stars: chemically peculiar - binaries: general

\section{Introduction}

So far, only a small number of planetary nebulae (PNe) have been identified to host a binary with a giant or subgiant component dominating the optical wavelength range and showing peculiar surface element abundances that indicate late stage stellar evolution nuclear synthesis. These stars exhibit signatures of slow neutron-capture nucleosynthesis (s-process) in their spectra and in some cases an enrichment in carbon (C).

For the object of this work, the central star (CS) of the PN Hen 2-39 (PN G283.8-04.2, Wray 16-64; Henize 1967; Acker et al. 1992; Wray 1966), Miszalski et al. (2013a) determined an overabundance for the s-process element barium (Ba) of $[\mathrm{Ba} / \mathrm{Fe}]^{1}=1.5 \pm 0.25$ and an enrichment of $[\mathrm{C} / \mathrm{H}]=$ $0.42 \pm 0.02$ in a spectral analysis based on mid-resolution spectra obtained with the Southern African Telescope (SALT; Buckley et al. 2006) with the Robert Stobie Spectrograph (RSS;

\footnotetext{
${ }^{\star}$ Based on data products from observations made with ESO Telescopes at the La Silla Paranal Observatory under program ID 093.D-0332(A).

$1 \quad[\mathrm{~A} / \mathrm{B}]=\log \left(n_{\mathrm{A}} / n_{\mathrm{B}}\right)-\log \left(n_{\mathrm{A}, \odot} / n_{\mathrm{B}, \odot}\right)$ with the number fractions $n$ for element $\mathrm{A}$ and $\mathrm{B}$.
}

Burgh et al. 2003; Kobulnicky et al. 2003). These findings confirm the membership of the K-type nucleus of Hen 2-39 in the small group of Ba central stars of planetary nebulae (CSPNe) along with LoTr 5, WeBo 1, and Abell 70 (Thevenin \& Jasniewicz 1997; Bond et al. 2003; Miszalski et al. 2012; Tyndall et al. 2013; Aller et al. 2018).

$\mathrm{Ba}$ CSPNe are prime examples of progenitors of Ba stars that were described by Bidelman \& Keenan (1951). Because of their evolutionary status, namely still being on the main sequence or a red giant, these stars did not yet experience AGB nucleosynthesis and, thus, cannot have synthesized heavy elements. McClure et al. (1980) discovered the binary nature of $\mathrm{Ba}$ stars and proposed that mass transfer was key to explain these sources. Boffin \& Jorissen (1988) performed detailed simulations of wind mass transfer to explain the pollution of the Ba star from an evolved companion with the products of asymptotic giant branch (AGB) nucleosynthesis that are dredged up to the stellar surface (Herwig 2005; Werner \& Herwig 2006). More recently, other mechanisms were proposed in which the material is transferred to the still unevolved companion (Boffin 2015) via Roche-lobe overflow (RLOF; e.g. Han et al. 1995) or wind-RLOF (e.g. Nagae et al. 2004; Mohamed \& Podsiadlowski 2007; Abate et al. 2013). 
This scenario is strongly supported by the fact that, so far, all Ba stars are found in binaries with white dwarf (WD) companions (McClure et al. 1980; McClure 1983; Jorissen \& Mayor 1988; McClure \& Woodsworth 1990; Jorissen et al. 1998), which is also definitely clear for the Ba CSPNe since the Ba star is not hot enough to ionize the ambient ejected material that is visible as the surrounding PN. Although the (pre-)WD companion must be there without any doubt, it can be difficult to detect against the bright companion even in the UV. Recently, more and more WD companions of Ba stars (Gray et al. 2011) and pre-WD companions of Ba CSPNe have been detected (e.g. Abell 70; Miszalski et al. 2012), which doubtlessly confirms the formation scenario. The still poorly understood mechanism of mass transfer in these systems is subject of ongoing research. The challenge is to determine their orbital parameters, such as eccentricity and period and to reproduce these with theoretical binary evolution models (Saladino et al. 2018; De Marco 2009).

These stars are expected to show orbital periods of several hundred days, which are typical values for Ba stars (Jorissen et al. 1998). However, there is the CSPN binary in the Necklace Nebula (PN G054.6-03.4; Corradi et al. 2011) standing out toward shorter periods. Miszalski et al. (2013b) found a period of $1.16 \mathrm{~d}$ for the post-CE system from the analysis of the C-dwarf secondary. On the other side of the period range, current analyses also indicate that there are systems with values up to several years and with larger eccentricities (Jones et al. 2017).

$\mathrm{Ba}$ CSPNe are ideal to study AGB nucleosynthesis. They provide a snapshot of an evolutionary stage with ideal conditions for analyzing not only the polluted cool (sub)giant star but also the ejected material of the nebula around the polluting post-AGB star (e.g. Madonna et al. 2017, 2018). The short duration of the PN phase $\left(\approx 10^{4} \mathrm{yr}\right)$ guarantees that the mass transfer happened recently and that the companion has not yet had time to adjust. Also, in some cases, the polluted star is still unevolved and did not experience the first dredge-up (DU) that would affect the surface element composition including the nucleosynthesis outcomes from the polluting post-AGB star.

By comparing the results of our comprehensive spectral analysis to theoretical AGB nucleosynthesis models (Karakas \& Lugaro 2016; Karakas et al. 2018), new insights into Ba stars and PNe are gained. It is worth mentioning the s-process mechanisms including atomic reaction rates, the source of neutrons and neutron exposure, internal stellar structures, and mixing processes occurring in a thermal-pulsing AGB star. This allows us to constrain the progenitor mass of the post-AGB star and the number of thermal pulses (TPs) on the AGB. Including binary evolution models (Saladino et al. 2018; De Marco 2009), these objects offer the opportunity to study the CE process and (wind-) RLOF, which are still far from being understood (Miszalski et al. 2013b; Jones \& Boffin 2017), and in addition the fraction of mass transferred (Boffin \& Jorissen 1988) and, following from this, the dilution factor in the Ba star itself and, thus, the mixing processes at work in (sub)giant stars (Husti et al. 2009). In particular, Ba CSPNe such as Hen 2-39 offer the possibility to detect technetium ( $\mathrm{Tc}$ ), which is the lightest element with no stable isotopes, in their atmospheres. This element was first detected by Merrill (1952) in the atmospheres of red giants, which proved that it is synthesized in evolved stars, since the half-life of ${ }^{99} \mathrm{Tc}$ of $210000 \mathrm{yr}^{2}$ is much shorter than the previous giant evolutionary phase. It is thus only observed in AGB stars currently undergoing thermal pulses (TPs; Van Eck \& Jorissen 1999;

\footnotetext{
2 Los Alamos National Laboratory Periodic Table http:// periodic.lanl.gov
}

Lebzelter \& Hron 2003) and, hence, the determination of the Tc surface abundance of the Ba CSPN indicates the mass-transfer link between the binary components in the PN and establishes a definite indicator for the existence of the third dredge-up (TDU). Assuming a typical post-AGB age of some $10^{3}-10^{4} \mathrm{yr}$ (Miller Bertolami 2016) for the primary component and taking into account that the dynamical process of mass transfer is short compared to this number (Iben \& Livio 1993; Chen et al. 2017), a large fraction of the transferred Tc should still be present in the stellar atmosphere.

We describe the observations, stellar atmosphere models, and analysis techniques in Sects. 2 and 3, respectively. The spectral analysis follows in Sects. 4 and 5. The results are discussed in Sect. 6. We summarize and conclude in Sect. 7.

\section{Observations}

The spectral analysis of Hen 2-39 is based on spectra in the optical wavelength range obtained with the Ultraviolet and Visual Echelle Spectrograph (UVES; Dekker et al. 2000) at the Very Large Telescope (VLT) at the Paranal Observatory of the European Southern Observatory under ESO program 093.D-0332(A). The data products created from this data were retrieved from the ESO Science Archive Facility. The observation $\log$, including the signal-to-noise ratio $(\mathrm{S} / \mathrm{N})$, of the spectra used in this paper is shown in Table A.1. All spectra were taken with a resolving power of $R=42000-44000$.

We used the spectral analysis code ISpec (Blanco-Cuaresma et al. 2014) to determine the radial velocities for each single observation via cross-correlation with a model template spectrum created using the fundamental parameters $T_{\text {eff }}, \log g$, $\mathrm{C}$ abundance, and metallicity determined by Miszalski et al. (2013a). The heliocentric corrected radial velocities for the 18 observations are given in Table 1 . To improve the $\mathrm{S} / \mathrm{N}$, all observations were shifted to the rest-frame velocity and subsequently co-added. To simulate the resolution of the instrument, all synthetic spectra shown in this work were convolved with Gaussians (full width half maximum $(F W H M)=0.12 \AA$ ).

\section{Model atmospheres, atomic data, and analysis techniques}

We used the stellar synthesis code SPECTRUM ${ }^{3}$ (Gray \& Corbally 1994, version 2.76) to calculate synthetic spectra for the analysis of the observed high-resolution spectra with the ATLAS9 model atmosphere grids ${ }^{4}$ (Kurucz 1991; Castelli \& Kurucz 2003) as input. These one-dimensional models are based upon the solar abundances from Grevesse \& Sauval (1998) and are calculated under the presumption of plane-parallel geometry and local thermodynamic equilibrium (LTE), which is valid for stars in this temperature and gravity regime (Hubeny et al. 2003). For the wavelength values and oscillator strengths of the lines selected in our analysis, we used the values provided within the distribution of SPECTRUM. Data for Tc I were retrieved from the Atomic Spectra Database ${ }^{5}$ of the National Institute of Standards and Technology (NIST). For Tc II, we used the data provided by Palmeri et al. (2007). We calculated an extensive grid of synthetic spectra spanning from $T_{\mathrm{eff}}=3500$ to $6000 \mathrm{~K}$

\footnotetext{
3 http://www .appstate. edu/ grayro/spectrum/spectrum html

4 http://kurucz.harvard.edu/grids.html

5 https://www.nist.gov/pml/atomic-spectra-database
} 
Table 1. Observation time and heliocentric radial velocities for the 18 observations of the Ba CSPN of Hen 2-39.

\begin{tabular}{lc}
\hline \hline MJD & $v_{\text {rad }}$ \\
\hline 56750.117 & $44.79 \pm 1.06$ \\
56750.135 & $44.76 \pm 1.01$ \\
56750.154 & $46.61 \pm 1.00$ \\
56750.172 & $45.64 \pm 1.09$ \\
56751.032 & $46.05 \pm 0.93$ \\
56751.050 & $46.47 \pm 0.92$ \\
56751.070 & $46.34 \pm 1.07$ \\
56751.088 & $46.29 \pm 1.03$ \\
56751.109 & $45.35 \pm 1.05$ \\
56751.127 & $45.17 \pm 1.05$ \\
56760.108 & $45.43 \pm 0.94$ \\
56760.126 & $45.56 \pm 1.06$ \\
56762.018 & $45.79 \pm 1.09$ \\
56762.035 & $45.21 \pm 1.00$ \\
56762.053 & $45.64 \pm 1.06$ \\
56762.070 & $45.91 \pm 1.08$ \\
56762.088 & $45.96 \pm 1.10$ \\
56762.106 & $46.24 \pm 1.09$
\end{tabular}

$\left(\Delta T_{\text {eff }}=250 \mathrm{~K}\right.$ between 4000 and $5000 \mathrm{~K}$ and $\Delta T_{\text {eff }}=500 \mathrm{~K}$ otherwise) and from $\log g\left(\mathrm{~cm}^{-1} \mathrm{~s}^{-2}\right)=0.0$ to $4.0(\Delta \log g=0.5)$ with a metallicity of $[\mathrm{M} / \mathrm{H}]=-0.3$ around the literature values of $T_{\text {eff }}=4250 \pm 150 \mathrm{~K}$ and $\log g=2.0 \pm 0.5$ (Miszalski et al. 2013a). For the determination of abundances, we relied on the model with $T_{\text {eff }}=4250 \mathrm{~K}$ and $\log g=2.5$ and varied the abundance of one single element over a range of at least 2.5 dex with a step of $0.5 \mathrm{dex}$. The exceptions to this are $\mathrm{C}$, for which we varied the abundance in steps of 0.05 dex over a range of $0.25 \mathrm{dex}$, and $\mathrm{N}$ with a range of $1.5 \mathrm{dex}$ and steps of $0.3 \mathrm{dex}$. Since the spectrum is crowded with absorption lines that are broadened by rotation, we could not measure equivalent widths to determine the fundamental parameters. We performed the analysis of the different parameters by selecting wavelength regions that show a strong influence of these particular species. The final values were then derived using a $\chi^{2}$-method applied to the synthetic spectra grid for the selected regions.

\section{Stellar parameters}

\subsection{Rotation}

To determine $v_{\text {rot }} \sin i=38 \pm 5 \mathrm{~km} \mathrm{~s}^{-1}$, we used a fit of a synthetic spectrum calculated with the literature values given by Miszalski et al. (2013a) and convolved with rotational profiles for values from $v_{\text {rot }} \sin i=0$ to $50 \mathrm{~km} \mathrm{~s}^{-1}$ to two regions spanning from 6440 to $6515 \AA$ and from 7030 to $7070 \AA$ dominated by strong $\mathrm{C}_{2}$ molecular absorption bands (Fig. 1).

\subsection{Effective temperature and surface gravity}

The many rotationally broadened lines in the observation also hamper the determination of equivalent widths. Thus, we used a set of diagnostic lines of Fe I, Fe II, Ti I, Ti II, Sc I, Sc II, and Mg I and performed a $\chi^{2}$-fit for selected wavelength regions. The set is composed of the lines that Tabernero et al. (2018) used for a similar spectral analysis. Furthermore, we included some Fe I and Fe II lines that are used by the Brussels Automatic Code for

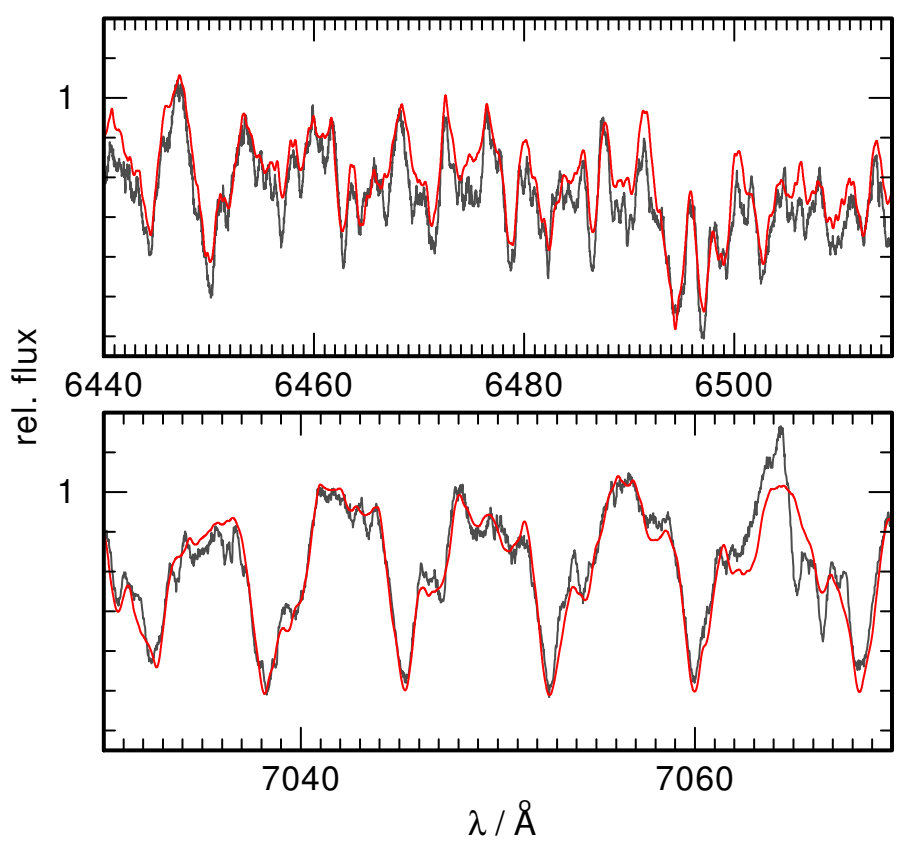

Fig. 1. Synthetic spectra (red) convolved with a rotational profile of $v_{\text {rot }}=38 \mathrm{~km} \mathrm{~s}^{-1}$ around strong $\mathrm{C}_{2}$ and $\mathrm{CN}$ molecular absorption bands compared with observations (gray).

Characterizing High accuracy Spectra (BACCHUS ${ }^{6}$; Masseron et al. 2016). Finally, we added lines for Fe I, Fe II, Ti I, Ti II, Sc I, and Sc II for which we obtained the largest theoretical equivalent widths in the calculation of the synthetic spectra and that did not saturate. The resulting collection of wavelength regions used for the analysis and the list of diagnostic lines is shown in Table A.2. They cover a wide range of different excitation potentials and oscillator strengths. After a first determination of $T_{\text {eff }}$ and $\log g$, we found a degeneracy in $\log g$ due to the fact that the strength of the computed lines in the regime around the literature values varies very little as a function of $\log g$ for a fixed value of $T_{\text {eff }}$. A spectroscopic determination of $\log g$ is hampered by uncertain values for the distance and brightness (Sect. 6.3). Thus, we adopt a value of $\log g=2.5 \pm 0.5$ that is typical for Ba giants of that type (e.g. de Castro et al. 2016). This approach seems to be reasonable because a change of $\Delta \log g=0.5$ only marginally affects the derived abundances compared to the significant statistical errors. We derive $T_{\text {eff }}=(4350 \pm 150) \mathrm{K}$. Figures A.1 and A.2 illustrate the spectroscopic determination of these parameters by showing the difference due to a variation of $T_{\text {eff }}$ and $\log g$.

\section{Element abundances}

We used model atmospheres with $T_{\text {eff }}=4250 \mathrm{~K}$ and $\log g=2.5$ from the grid and performed line-profile fits for the following elements to determine their abundances. The results are given in Table 2. In our analysis, we assumed the atomic data to be correct and did not propagate uncertainties on atomic data. The continuum placement uncertainty is also assumed to be small as the continuum placement is shifted during the fitting procedure. To estimate the impact of a varied $T_{\text {eff }}$ and $\log g$ on the determined abundances, we redid part of our analysis with models with varied $T_{\text {eff }}$ between 4000 and $4500 \mathrm{~K}$ at $\log g$ varied between 2.0 and 3.0. We also varied the microturbulence

6 http://www.astro.ulb.ac.be/pmwiki/Spectro/Bacchus 
Table 2. Element abundances determined for Hen 2-39 in $\log \epsilon=$ $12+\log \left(n_{\mathrm{X}} / n_{\mathrm{H}}\right),[\mathrm{X} / \mathrm{H}]=\log \left(n_{\mathrm{X}} / n_{\mathrm{H}}\right)-\log \left(n_{\mathrm{X}, \odot} / n_{\mathrm{H}, \odot}\right)$, and $[\mathrm{X} / \mathrm{Fe}]=$ $\log \left(n_{\mathrm{X}} / n_{\mathrm{Fe}}\right)-\log \left(n_{\mathrm{X}, \odot} / n_{\mathrm{Fe}, \odot}\right)$ with the number fraction $n_{\mathrm{X}}$ for element X.

\begin{tabular}{|c|c|c|c|c|}
\hline Element & $\log \epsilon$ & {$[\mathrm{X} / \mathrm{H}]$} & {$[\mathrm{X} / \mathrm{Fe}]$} & Error \\
\hline $\mathrm{C}$ & 8.9 & 0.36 & 0.71 & 0.08 \\
\hline $\mathrm{N}$ & 8.3 & 0.3 & 0.7 & 0.8 \\
\hline $\mathrm{Na}$ & 6.0 & -0.3 & 0.1 & 0.7 \\
\hline $\mathrm{Al}$ & 5.8 & -0.7 & -0.3 & 1.0 \\
\hline S & 8.1 & 0.8 & 1.2 & 1.2 \\
\hline $\mathrm{K}$ & 4.8 & -0.3 & 0.1 & 1.0 \\
\hline $\mathrm{Ca}$ & 5.9 & -0.4 & -0.1 & 1.0 \\
\hline $\mathrm{Sc}$ & $<2.4$ & $<-0.7$ & $<-0.4$ & \\
\hline $\mathrm{Ti}$ & 4.0 & -0.9 & -0.6 & 1.4 \\
\hline V & 3.1 & -0.9 & -0.6 & 1.0 \\
\hline $\mathrm{Cr}$ & 5.1 & -0.5 & -0.2 & 1.3 \\
\hline $\mathrm{Mn}$ & 5.1 & -0.3 & 0.0 & 1.0 \\
\hline $\mathrm{Fe}$ & 7.1 & -0.3 & & 1.0 \\
\hline $\mathrm{Co}$ & 5.1 & 0.2 & 0.5 & 1.0 \\
\hline $\mathrm{Ni}$ & 6.5 & 0.2 & 0.6 & 1.3 \\
\hline $\mathrm{Cu}$ & 5.0 & 0.8 & 1.2 & 1.5 \\
\hline $\mathrm{Zn}$ & $<5.8$ & $<1.2$ & $<1.6$ & \\
\hline $\mathrm{Rb}$ & 3.7 & 1.1 & 1.4 & 1.3 \\
\hline $\mathrm{Sr}$ & 3.6 & 0.6 & 1.0 & 1.5 \\
\hline $\mathrm{Y}$ & 2.3 & 0.0 & 0.4 & 1.5 \\
\hline $\mathrm{Zr}$ & 2.4 & -0.2 & 0.2 & 1.5 \\
\hline $\mathrm{Nb}$ & $<2.0$ & $<0.7$ & $<1.0$ & \\
\hline Mo & 2.9 & 1.0 & 1.4 & 1.3 \\
\hline $\mathrm{Tc}$ & $<2.5$ & & & \\
\hline $\mathrm{Ru}$ & $<3.5$ & $<1.7$ & $<2.1$ & \\
\hline $\mathrm{Ba}$ & 3.6 & 1.4 & 1.8 & 0.5 \\
\hline $\mathrm{La}$ & 2.3 & 1.1 & 1.5 & 1.6 \\
\hline $\mathrm{Ce}$ & $<3.5$ & $<2.0$ & $<2.3$ & \\
\hline $\operatorname{Pr}$ & $<3.0$ & $<2.4$ & $<2.7$ & \\
\hline $\mathrm{Nd}$ & 1.9 & 0.4 & 0.8 & 1.5 \\
\hline $\mathrm{Sm}$ & $<1.7$ & $<0.8$ & $<1.1$ & \\
\hline $\mathrm{Eu}$ & $<1.1$ & $<0.6$ & $<1.0$ & \\
\hline $\mathrm{Gd}$ & $<2.6$ & $<1.5$ & $<1.8$ & \\
\hline $\mathrm{Tb}$ & $<0.8$ & $<0.5$ & $<0.8$ & \\
\hline Dy & $<4.5$ & $<3.4$ & $<3.8$ & \\
\hline $\mathrm{Er}$ & $<2.4$ & $<1.6$ & $<1.9$ & \\
\hline $\mathrm{Hf}$ & $<1.8$ & $<1.0$ & $<1.4$ & \\
\hline W & 1.4 & 0.7 & 1.1 & 1.5 \\
\hline Os & $<2.9$ & $<1.5$ & $<1.8$ & \\
\hline
\end{tabular}

velocity by $\pm 2.0 \mathrm{~km} \mathrm{~s}^{-1}$, which was kept fixed at $2.0 \mathrm{~km} \mathrm{~s}^{-1}$ in the initial analysis. By far, the impact of a change in temperature is the largest. Compared to this error, the variation in $\log g$ and microturbulence velocity become negligible. Furthermore, we investigated the influence of the metallicity of the model atmosphere grid that was chosen for the analysis on the determined abundances and repeated part of the analysis with different input model metallicities between -0.5 and 0 . Raising (lowering) the metallicity by a certain amount results in a $\mathrm{Fe}$ abundance that is lower (higher) by roughly 1.3 -fold that amount. Consistency is reached for the grid with $[\mathrm{M} / \mathrm{H}]=-0.3$ that gives a $\mathrm{Fe}$ abundance of $[\mathrm{Fe} / \mathrm{H}]=0.3 \pm 1.0$. In our analysis, we find a $\mathrm{C}$ enrichment but cannot determine the $\mathrm{O}$ abundance and, thus, use the solar value. The resulting $\mathrm{C} / \mathrm{O}$ ratio is larger than one. To test whether it is justified to use an O-rich model atmosphere grid (model 1) with solar abundances, we employed the ATLAS9

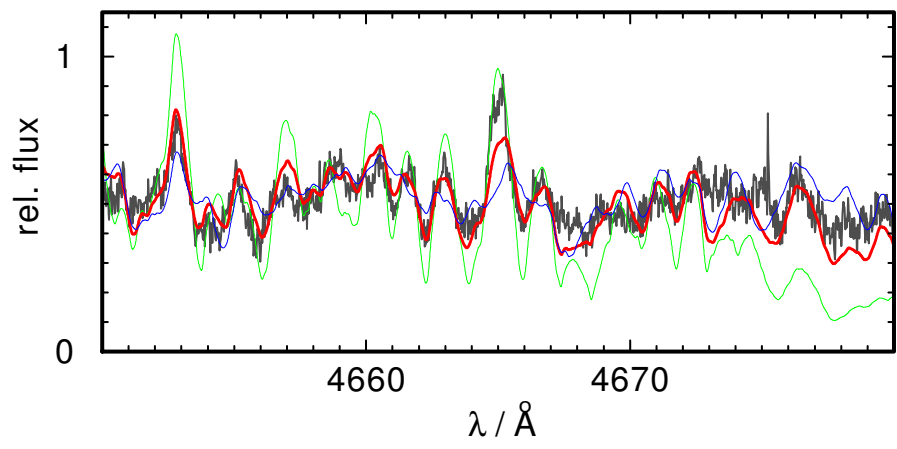

Fig. 2. Observation (gray) of Hen 2-39 compared to model spectra with $T_{\text {eff }}=4250 \mathrm{~K}$ and $\log g=2.5$ for a selected region of strong $\mathrm{C}_{2}$ absorption for $[\mathrm{C} / \mathrm{H}]=0.46,0.36,0.26$ (green, red, and blue, respectively).

code and calculated a test model with a $\mathrm{C}$ abundance increased to the value found in our analysis (model 2). Furthermore, we calculated a second test model with the increased $\mathrm{C}$ abundance and an $\mathrm{O}$ abundance reduced by one dex (model 3 ). We redid the abundance analysis for $\mathrm{C}$ and $\mathrm{Ba}$ and found the same abundance for $\mathrm{C}$ from model 1 and 2 . For the model with increased $\mathrm{C}$ and decreased $\mathrm{O}$ abundance, we get a $\mathrm{C}$ abundance that is 0.03 dex higher. Compared to model 1 , the $\mathrm{Ba}$ abundance for model 2 is higher by 0.04 dex and by 0.2 dex for model 3 . The difference between the O-rich model 1 and the C-rich model 2 is shown in Fig. A.3. The effect of the model atmosphere on the abundances is in a range that justifies relying on the available model atmosphere grid. Thus, we did not compute a $\mathrm{C}$-rich grid. However, this adds another uncertainty to the abundances. We obtain large errors arising from the crowdedness of the observed spectrum that we estimate by detailed line profile fits and evaluation based on the $\chi$-by-eye method. In many cases, this is the main contributor to the total error. It ranges between 0.05 dex for $\mathrm{C}$ and about $0.4 \mathrm{dex}$ for the light metals up to about $1 \mathrm{dex}$ for the iron group and trans-iron elements. To take the uncertainties in $T_{\text {eff }}$ and $\log g$ into account, we did this procedure for the corners of the grid stated above $\left(T_{\text {eff }}=4000 \mathrm{~K}, \log g=3.0\right.$ and $4500 \mathrm{~K}, 2.0)$. The abundance errors arising from this effect range between 0.03 dex for $\mathrm{C}$ and about $0.5 \mathrm{dex}$ for the other metals. The total errors given in Table 2 are the maximum differences for the abundances that are possible within the error limits of this grid.

Carbon. We analyzed the $\mathrm{C}$ abundance using spectrum synthesis calculations for the region of strong $\mathrm{C}_{2}$ absorption from 4650 to $4737 \AA$ (Fig. 2). We confirm the $\mathrm{C}$ enhancement and our result of $[\mathrm{C} / \mathrm{H}]=0.36 \pm 0.08$ agrees within $1 \sigma$ with the value derived by Miszalski et al. (2013a) from their mid-resolution spectra.

Nitrogen. Using the $\mathrm{C}$ abundances, we derived the $\mathrm{N}$ abundance from synthetic calculations for the wavelength regions 7030-7070 $\AA$ and 7900-8100 $\AA$ affected by strong CN absorption bands. Figure 3 shows the best result. We find $\mathrm{N}$ to be enriched to the same level as $\mathrm{C}$ with $[\mathrm{N} / \mathrm{H}]=0.3 \pm 0.8$. We could not identify any line of oxygen in the observed spectrum and, thus, were unable to fix an abundance value for $\mathrm{O}$. In our analysis, we adopt the solar value. To get an idea of the ${ }^{12} \mathrm{C} /{ }^{13} \mathrm{C}$ ratio, we analyzed the $\mathrm{CN}$ absorption band in the region $8100-8200 \AA$ and included the line list for ${ }^{13} \mathrm{C}^{14} \mathrm{~N}$ from Sneden et al.(2014; Fig. 4). From the inspection of the observation, we cannot claim to find a enhancement in ${ }^{13} \mathrm{C}$ resulting in 


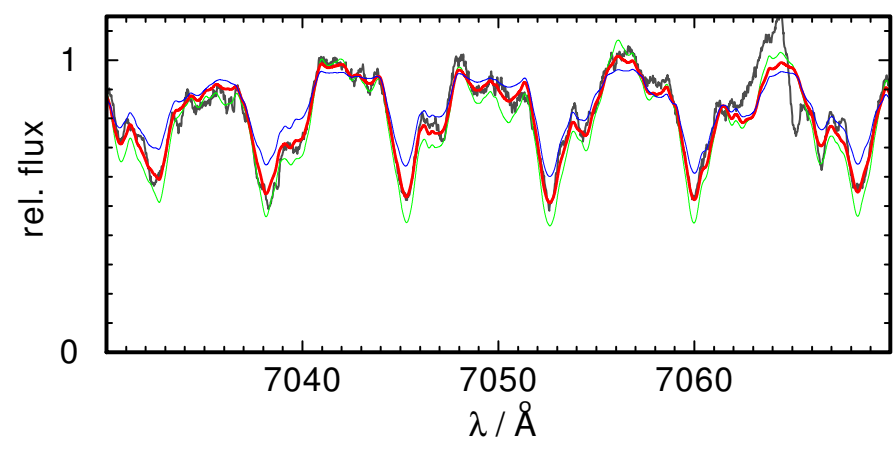

Fig. 3. Like Fig. 2, for strong $\mathrm{CN}$ absorption for $[\mathrm{N} / \mathrm{H}]=1.1,0.3,-0.5$ (green, red, and blue, respectively).

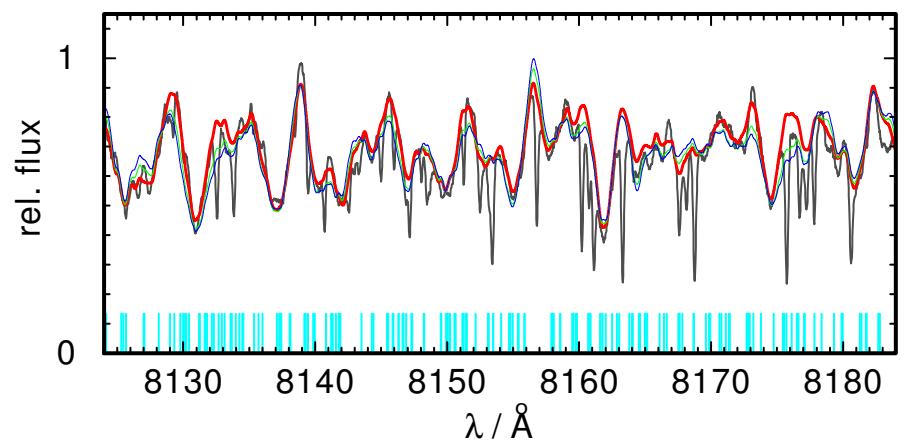

Fig. 4. Like Fig. 2, for strong $\mathrm{CN}$ absorption for ${ }^{12} \mathrm{C} /{ }^{13} \mathrm{C}=90,10,5$ (red, green, and blue, respectively). ${ }^{13} \mathrm{CN}$ absorption lines are indicated at the bottom in cyan.

${ }^{12} \mathrm{C} /{ }^{13} \mathrm{C}$ lower than the solar value of 90 , although this cannot be ruled out.

Light metals: sodium to potassium. For the following elements, we used the most prominent absorption features in the synthetic spectra, which show the largest impact of a change of the abundance (Table A.3). The Na doublet Na II $\lambda \lambda 5890.8,5896.5 \AA$ (Fig. A.4) is used to find a Na abundance of $[\mathrm{Na} / \mathrm{Fe}]=0.1 \pm 0.7$, which resembles well with the sample of Ba stars analyzed by de Castro et al. (2016, Fig. 6). From a fit to the regions affected by absorption due to $\mathrm{Al}$ we derive $[\mathrm{Al} / \mathrm{Fe}]=-0.3 \pm 1.0$. Ba stars typically show a slight enhancement in $\mathrm{Al}$, which is also present in the sample of de Castro et al. (2016). For $\mathrm{S}$ we derive $[\mathrm{S} / \mathrm{Fe}]=1.2 \pm 1.2$ and for $\mathrm{K}$ we found $[\mathrm{K} / \mathrm{Fe}]=0.1 \pm 1.0$.

Iron-peak elements: calcium to copper. Selected absorption features due to neutral $\mathrm{Ca}$ to $\mathrm{Cu}$ are shown in Figs. A.5 and A.6. We performed a fit for a set of Fe I and Fe II absorption lines and found this star to be metalpoor with $[\mathrm{Fe} / \mathrm{H}]=-0.3 \pm 1.0$. For all iron-peak elements prior to $\mathrm{Fe}$, we found solar values or slight underabundances, namely $[\mathrm{Ca} / \mathrm{Fe}]=-0.1 \pm 1.0,[\mathrm{Ti} / \mathrm{Fe}]=-0.6 \pm 1.4,[\mathrm{~V} / \mathrm{Fe}]=$ $-0.6 \pm 1.0,[\mathrm{Cr} / \mathrm{Fe}]=-0.2 \pm 1.3$, and $[\mathrm{Mn} / \mathrm{Fe}]=0.0 \pm 1.0$. For $\mathrm{Sc}$, we determined an upper limit of $[\mathrm{Sc} / \mathrm{Fe}]=-0.4$. These low values for the $\alpha$ elements $\mathrm{Ca}$ and $\mathrm{Ti}$ do not correspond with the trend in the sample of de Castro et al. (2016) who found an enrichment of these elements compared to $\mathrm{Fe}$ with decreasing metallicity. The values close to solar for the other elements, however, are in good agreement with the sample showing a clustering of the abundances around $[\mathrm{X} / \mathrm{Fe}]=0.0$. For the elements of this group subsequent to $\mathrm{Fe}$, we determined overabundances compared to $\mathrm{Fe}$ of $[\mathrm{Co} / \mathrm{Fe}]=0.5 \pm 1.0,[\mathrm{Ni} / \mathrm{Fe}]=0.6 \pm 1.3$, and $[\mathrm{Cu} / \mathrm{Fe}]=1.2 \pm 1.5$.

Trans-iron elements: zinc to osmium. Selected wavelength regions that are among those showing the largest impact of a change in abundance for the elements of this group are shown in Figs. A.7-A.9. Since we found only few significant absorption features for the majority of these elements, the statistical errors of the determined values are large (often $>1$ dex) and in many cases only upper abundance limits could be established. We obtained $[\mathrm{Zn} / \mathrm{Fe}]<1.6$ and $[\mathrm{Rb} / \mathrm{Fe}]=1.4 \pm 1.3$. For the elements around the first peak of the s-process, we found $[\mathrm{Sr} / \mathrm{Fe}]=$ $1.0 \pm 1.5,[\mathrm{Y} / \mathrm{Fe}]=0.4 \pm 1.5$, and $[\mathrm{Zr} / \mathrm{Fe}]=0.2 \pm 1.5$. Despite the large uncertainties, we find a good agreement with the $\mathrm{Sr}$ abundances of the sample of Karinkuzhi et al. (2018, Fig. 6). The $\mathrm{Sr}$ abundances for most of the Ba stars of Merle et al. (2016) are slightly lower but some reach up to 1 dex as well. Also the $\mathrm{Y}$ abundance of Hen 2-39 agrees well with the sample of Merle et al. (2016), whereas the majority of Ba giants of Karinkuzhi et al. (2018) and de Castro et al. (2016) crowd around higher values of $\mathrm{Y}$. The $[\mathrm{Zr} / \mathrm{Fe}]$ abundances of Karinkuzhi et al. (2018) are significantly higher (all $>1 \mathrm{dex}$ ), whereas some of the Ba stars of Merle et al. (2016) agree with low values of $[\mathrm{Zr} / \mathrm{Fe}]$. Also the sample of de Castro et al. (2016) clusters around $[\mathrm{Zr} / \mathrm{Fe}] \approx 1$ but also shows stars with comparatively low values like that for Hen $2-39$. For the elements subsequent to this peak, we determined $[\mathrm{Nb} / \mathrm{Fe}]<1.0,[\mathrm{Mo} / \mathrm{Fe}]=1.4 \pm 1.3$, and $[\mathrm{Ru} / \mathrm{Fe}]<2.1$.

One key element of this analysis is the radioactive Tc. The strongest absorption features that appear in the synthetic spectra are Tc I $\lambda \lambda 4031.6,4095.7,4238.2,4262.3,4297.1 \AA$. Unfortunately, these lines could not be clearly identified in the observed spectrum but it could be used to establish an upper abundance limit of $\log \epsilon_{\mathrm{Tc}}<2.5^{7}$.

For the determination of the $\mathrm{Ba}$ abundance, we used Ba II $\lambda \lambda 4554.0,4931.1,5853.7,6141.7,6496.9 \AA$. The first two are very strong and sensitive to small variations of the abundance (Fig. A.8). This helped to constrain $[\mathrm{Ba} / \mathrm{Fe}]=1.8 \pm 0.5$, which agrees within the error limits with the previous value of Miszalski et al. (2013a). The values determined by Karinkuzhi et al. (2018) for this element range from $0.81 \leq[\mathrm{Ba} / \mathrm{Fe}] \leq 2.67$. Also the sample of Merle et al. (2016) shows a scatter between almost solar and 2.5. Our strong enrichment found for Hen 2-39, thus, is not exceptional (Fig. 6).

La II absorption lines yield $[\mathrm{La} / \mathrm{Fe}]=1.5 \pm 1.6$. For the other elements of the second peak of the s-process we found $[\mathrm{Ce} / \mathrm{Fe}]<2.3,[\mathrm{Pr} / \mathrm{Fe}]<2.7$, and $[\mathrm{Nd} / \mathrm{Fe}]=0.8 \pm 1.5$. The upper limit for Ce lies above the value range of $1.02 \leq[\mathrm{Ce} / \mathrm{Fe}] \leq$ 1.76 determined by Karinkuzhi et al. (2018) and also above that of Merle et al. (2016) ranging from solar to 1.5 dex. The Ba stars of de Castro et al. (2016) cluster around an enrichment of $1.0 \mathrm{dex}$ and none of these stars reach values above 2.5 dex. Our upper limit for Pr lies above the value range of $1.18 \leq[\mathrm{Pr} / \mathrm{Fe}] \leq 2.55$ of Merle et al. (2016). However, our value for the $\mathrm{Nd}$ abundance is below their range of values. The star with the lowest Nd abundance shows $[\mathrm{Nd} / \mathrm{Fe}]=1.18$, whereas the sample of de Castro et al. (2016) clustering around $[\mathrm{Nd} / \mathrm{Fe}] \approx 1.0$ perfectly agrees with our value within the error limits.

For the further rare-earth elements Sm, Eu, Gd, Tb, Dy, and Er, we could only establish upper abundance limits (Table 2). These elements are not analyzed by Merle et al. (2016) and de Castro et al. (2016). Karinkuzhi et al. (2018) found ranges

\footnotetext{
$7 \log \epsilon=12+\log \left(n_{\mathrm{X}} / n_{\mathrm{H}}\right)$.
} 
of $1.02 \leq[\mathrm{Sm} / \mathrm{Fe}] \leq 2.17,0.96 \leq[\mathrm{Eu} / \mathrm{Fe}] \leq 1.43$, and $1.60 \leq$ $[\mathrm{Dy} / \mathrm{Fe}] \leq 2.51$. Our upper limits of $[\mathrm{Sm} / \mathrm{Fe}]<1.1$, and $[\mathrm{Eu} / \mathrm{Fe}]<1.0$ lie within these ranges; $[\mathrm{Dy} / \mathrm{Fe}]<3.8$ is significantly higher. Furthermore, we could determine the abundances of $\mathrm{Hf}, \mathrm{W}$, and Os to be $[\mathrm{Hf} / \mathrm{Fe}]<1.4,[\mathrm{~W} / \mathrm{Fe}]=1.1 \pm 1.5$, and $[\mathrm{Os} / \mathrm{Fe}]<1.8$.

\section{Discussion}

\subsection{Element abundances}

We compared our results with the yields from nucleosynthesis calculations of Karakas \& Lugaro (2016) for a metallicity of $Z=0.007$, in line with the low metallicity of $Z=0.006$ that we determined from $Z=10^{[\mathrm{Fe} / \mathrm{H}]} Z_{\odot}$ with $[\mathrm{Fe} / \mathrm{H}]=-0.3$ and $Z_{\odot}=0.0134$ (Asplund et al. 2009). From these models and those of Karakas et al. (2018), it becomes obvious that AGB nucleosynthesis does not affect the abundances of the iron peak elements and, thus, it seems reasonable to assume the same low metallicity for both components of the binary.

The fact that we cannot see a ${ }^{13} \mathrm{C}$ enhancement agrees very well with the theoretical calculations predicting even an enhancement of the initial solar ${ }^{12} \mathrm{C} /{ }^{13} \mathrm{C}$-ratio for models with initial masses $1.5 M_{\odot} \leq M_{\text {ini }} \leq 4.0 M_{\odot}$ where our estimated initial mass (Sect. 6.2) lies within.

The finding that the iron-peak elements prior to $\mathrm{Fe}$ show underabundances and those subsequent to $\mathrm{Fe}$ are enhanced leads to the speculation that this pattern may be caused by neutron capture on the former elements as seed species and the formation of elements heavier than Fe. Figure 5 also shows an enrichment due to AGB nucleosynthesis for the elements subsequent to $\mathrm{Fe}$.

The observed $\mathrm{N}$ enhancement of $[\mathrm{N} / \mathrm{Fe}]=0.7 \pm 0.8$ (Fig. 5) is in line with the enhancement found for the $\mathrm{Ba}$ stars of Karinkuzhi et al. (2018, Fig. 6). A high [N/C] ratio as found for this object is discussed in the literature (e.g. Smiljanic et al. 2006; Merle et al. 2016). These authors argue that CN processing in Ba stars could result in higher $\mathrm{N}$ abundances. According to Smiljanic et al. (2006), an increased [N/C] ratio can be caused by mixing events such as the first DU or by a more complex mixing process due to rotation for intermediate mass stars. This would be an indicator for hydrogen burning via the $\mathrm{CNO}$-cycle in the stellar core. With the assumed mass for the primary star (Sect. 6.2), this should be the dominating fusion process in this star. The fast rotation of the Ba-CSPN is most likely due to transfer of angular momentum from the primary and therefore does not imply that this star was rotating exceptionally fast initially so as to affect its $[\mathrm{N} / \mathrm{C}]$ abundance ratio.

For Tc, we could not identify the presence of any line without doubt and, thus, cannot constrain the abundance further than $\log \epsilon_{\mathrm{Tc}}<2.5$. Therefore, we cannot claim this star to have Tc in its atmosphere, which would directly lead to the necessity of prior mass transfer. The models of Karakas \& Lugaro (2016) predicted a final surface abundance between $\log \epsilon_{\mathrm{Tc}}=1.11$ and 1.24 for the models with initial masses between 2.1 and $2.5 M_{\odot}$, which lies well below the upper limit for Hen 2-39. Another diagnostic element reflecting recent s-process nucleosynthesis is $\mathrm{Nb}$. According to Neyskens et al. (2015), this mono-isotopic species is synthesized by the decay of the radioactive ${ }^{93} \mathrm{Zr}$ produced by s-process nucleosynthesis. Compared to ${ }^{99} \mathrm{Tc}$, this species has a longer half-life time of 1.53 Myr. Following our estimate made for Tc (Sect. 1), we do not expect an significant enrichment in $\mathrm{Nb}$, since the primary's post-AGB age should be much shorter than the ${ }^{93} \mathrm{Zr}$ half-life and, thus, a large fraction of this species should still be present. Thus, the $\mathrm{Nb} / \mathrm{Zr}$ would not represent the
${ }^{93} \mathrm{Zr} / \mathrm{Zr}$ ratio at the end of the AGB and cannot be employed as proof for prior mass transfer. Furthermore, the $\mathrm{Zr}$ abundance can be determined only within a very large error range and for $\mathrm{Nb}$, we find an upper limit only.

The detection of $\mathrm{Tc}$ is not hampered by the resolution of the spectrograph. The limiting factor is the $\mathrm{S} / \mathrm{N}$. We estimate the needed $\mathrm{S} / \mathrm{N}$ that would be necessary to clearly distinguish between a model without $\mathrm{Tc}$ and one with $\log \epsilon_{\mathrm{Tc}}=1.2$. From Fig. 7, it becomes clear, that the current $\mathrm{S} / \mathrm{N}$ is not sufficient to determine a Tc abundance of that level. Currently, the single spectra have a $\mathrm{S} / \mathrm{N}$ of 3 at that wavelength region. This is increased by co-adding all the spectra, but still a $\mathrm{S} / \mathrm{N}$ increased by a factor of 3 would be necessary. According to the UVES exposure time calculator (ETC), the needed $\mathrm{S} / \mathrm{N}$ would require about a six fold longer exposure. For the future Extremely Large Telescope (ELT) the estimate is more promising. By using the E-ELT Spectroscopic ETC, we find that the required $\mathrm{S} / \mathrm{N}$ is reached with an exposure of about half that of a single observation used in this analysis. For stars with a lower rotational velocity, the detection would become easier (Fig. 7). Unfortunately, all $\mathrm{Ba}$ CSPNe that are known up to now seem to rotate fast (shortest period of $4.7 \mathrm{~d}$ for Abell 70 and WeBo 1 and longest period of 5.9 d for LoTr 5; Bond et al. 2003; Miszalski et al. 2012; Aller et al. 2018), most likely because of the transfer of angular momentum by accretion of matter from the companion.

\subsection{Mass transfer}

By comparing our determined enrichment in s-process elements to the yields from evolutionary models for different initial masses of Karakas et al. (2018), we try to confirm that this can be the result of realistic mass transfer. For a primary that is currently in the stage of a CSPN, the secondary should have a mass that is lower by about $5 \%$ to be currently in the evolutionary stage of a red giant (assuming a mass dependent relation for the main-sequence lifetime $t \sim M^{-2.5}$ ). According to Joss et al. (1987), these stars should have a radiative core of about $0.3 M_{\odot}$ and a convective envelope of a mass $M_{\text {env }}=M_{\text {ini }}-0.3 M_{\odot}$ within which the accreted mass becomes diluted. We want to determine a realistic mass range for the primary by comparing the total mass for the different elements that is ejected during the AGB evolution with the mass that the secondary would have needed to accrete to become that enriched. The mass of element $\mathrm{X}$ that needs to be accreted is given by $M_{\text {need }}=M_{\text {env }}\left(\mathrm{mf}_{\mathrm{X}, \text { final }}-\right.$ $\mathrm{mf}_{\mathrm{X} \text {,initial }}$ ) with a final mass fraction $\mathrm{mf}_{\mathrm{X} \text {,final }}$ according to our analysis results and an initial mass fraction $\mathrm{mf}_{\mathrm{X} \text {,initial }}$ according to the low metallicity. In Fig. 8, we show the needed mass compared to the total ejected mass for $\mathrm{C}, \mathrm{N}$, and the elements heavier than Fe that show a significant production due to AGB nucleosynthesis. Since most of the abundances could not be constrained within small error limits, we focus on the $\mathrm{C}$ and $\mathrm{Ba}$ abundances. For all models of Fig. 8 and some additional models, we calculated the percentage of the total ejecta that would need to be accreted to produce the observed enrichment (Table 3 ). It is obvious that only the models for an initial mass between 1.5 and 4.0 $M_{\odot}$ can explain the enrichment due to a realistic mass transfer. For the models with the lowest initial masses as well as for those with the highest masses, the ratio of the yield of $\mathrm{Ba}$ to that of $\mathrm{C}$ is smaller, i.e., these models produce a smaller amount of $\mathrm{Ba}$ compared to $\mathrm{C}$. The fraction of accreted mass of $\mathrm{C}$ and $\mathrm{Ba}$ should be equal. Thus, a 1.75-3.00 $M_{\odot}$ progenitor seems to be most consistent with the abundance determinations. For this analysis, we used the models with the largest ${ }^{13} \mathrm{C}$-pocket that are available 


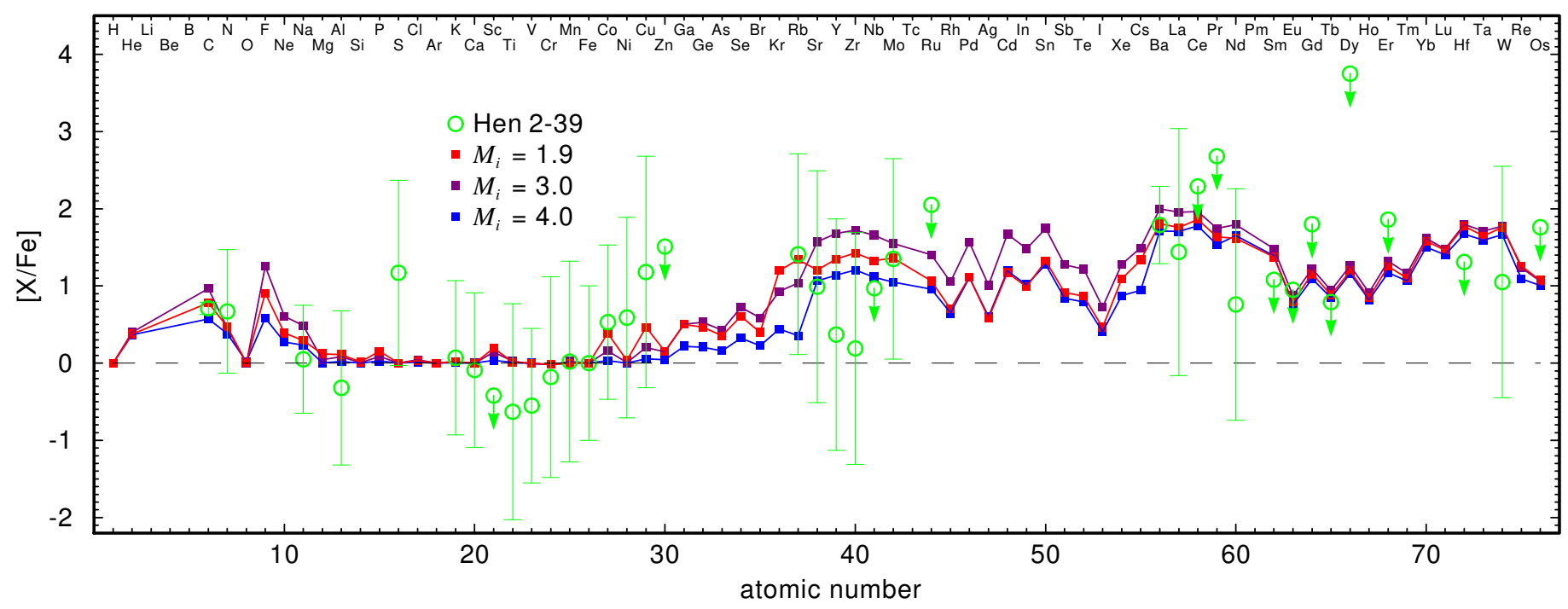

Fig. 5. Atmospheric element abundances of Hen 2-39 compared to the final yields of a selection of evolutionary models from Karakas \& Lugaro (2016) with a metallicity of $Z=0.007$. The initial masses are indicated in the upper panel. Arrows indicate upper limits.

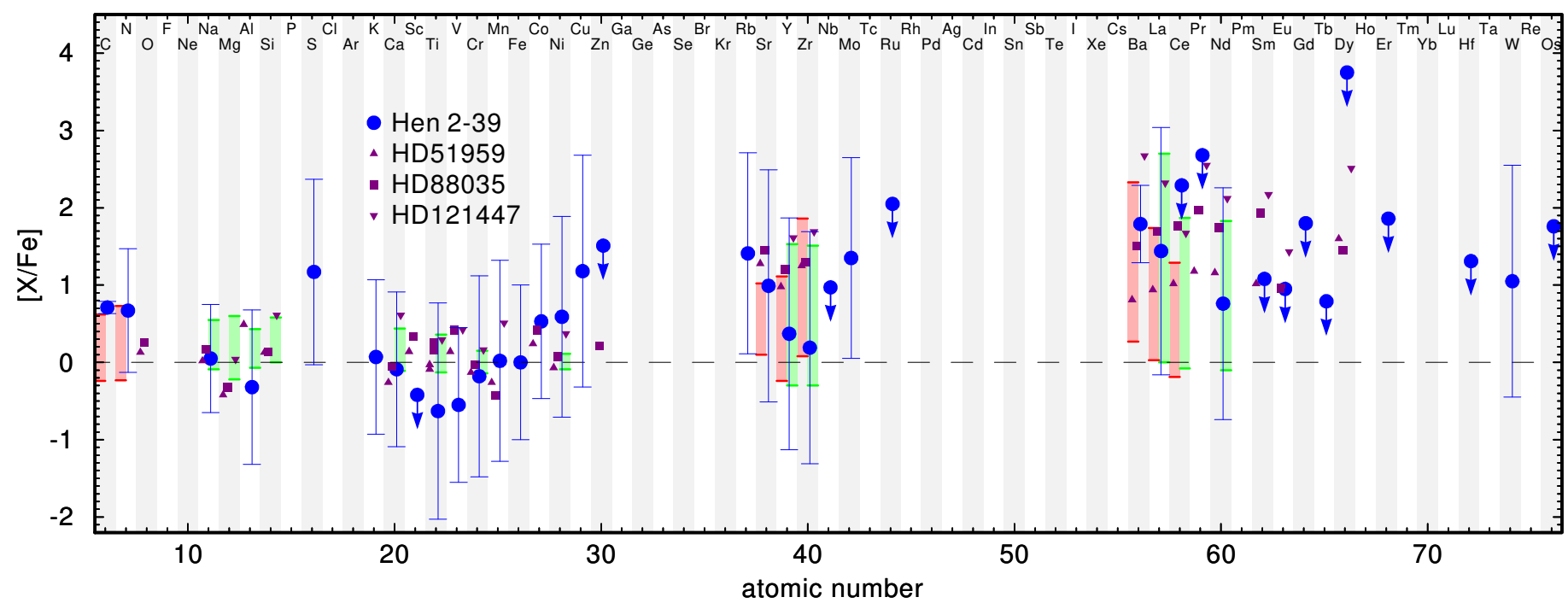

Fig. 6. Atmospheric element abundances of Hen 2-39 (blue) compared to the values for the three Ba stars HD515959, HD88035, and HD121447 analyzed by Karinkuzhi et al. (2018, purple) and the ranges that Merle et al. (2016, red) and de Castro et al. (2016, green) found for their sets of Ba-stars. Arrows indicate upper limits.

from Karakas \& Lugaro (2016). The larger the pocket size, the lower the yield of $\mathrm{C}$ and the higher that of $\mathrm{Ba}$, i.e., the ratio of the yield of $\mathrm{Ba}$ to that of $\mathrm{C}$ is larger. Even for the models with the largest ${ }^{13} \mathrm{C}$-pocket, the percentage of the total ejecta that would need to be accreted is higher considering the $\mathrm{Ba}$ abundance compared to the $\mathrm{C}$ abundance. This ratio becomes worse for smaller pocket sizes. In addition to the choice of the ${ }^{13} \mathrm{C}$-pocket, yields of the evolutionary models are affected by uncertainties due to mass loss, convective mixing, reaction rates, and neutron poisons (Karakas \& Lattanzio 2014). These effects are not evaluated by Karakas \& Lugaro (2016) and we take the tabulated yields without considering an error range. However, the progentitor mass estimate is affected by large uncertainties on the abundances and on the model yields and, thus, should be treated with caution.

This result leads to the conclusion that even such a high enrichment can reasonably be explained with realistic masstransfer mechanisms such as wind-RLOF (Chen et al. 2017). In this scenario only a small fraction of mass becomes unbound from the binary and the percentage of accreted mass ranges between 20 and $40 \%$. Simulations indicate that a binary with a wide separation, where mass transfer would act via the BondiHoyle mechanism, can be ruled out since the percentage of accreted mass decreases to only 2-3\% (Theuns et al. 1996). CE evolution would imply a short orbital period ( $\leq$ a few days), which is not the case for this binary and, thus, this scenario is also ruled out.

\subsection{Spectroscopic distance}

For Hen 2-39, several different distances are published. The nebular analysis of Miszalski et al. (2013a) yields $5.7 \mathrm{kpc}$. Frew et al. (2016) found $7.6_{-1.3}^{+1.5} \mathrm{kpc}$ based on the companion spectral type. The CSPN of Hen 2-39 is in the Gaia data release (DR2, ID: 5256396485463285504, Gaia Collaboration 2018). 


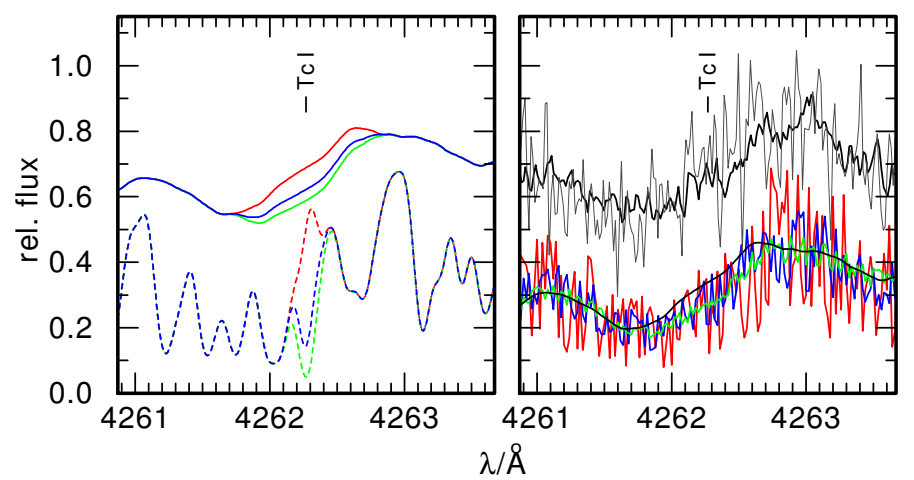

Fig. 7. Left panel: comparison between models with $\log \epsilon_{\mathrm{Tc}}=$ $0.0,1.2,2.5$ (red, blue, green) for models broadened with a rotational profile $\left(v_{\text {rot }}=36 \mathrm{~km} \mathrm{~s}^{-1}\right.$, solid lines with 0.35 offset $)$ and without rotation (dashed). Right panel: comparison between a rotationally broadened model without Tc (black) and models with $\log \epsilon_{\mathrm{Tc}}=1.2$ with different levels of artificial noise ( $\mathrm{S} / \mathrm{N}$ of the single observation: red, $\mathrm{S} / \mathrm{N}$ of co-added spectrum: blue, required $\mathrm{S} / \mathrm{N}$ : green). For comparison, the observation is shown with an offset of 0.35 (single spectrum: gray, co-added spectra: black).

Table 3. Percentage of the total ejecta that would need to be accreted to produce the observed enrichment of Hen 2-39 for evolutionary models of Karakas \& Lugaro (2016) with $Z=0.007$ for different initial masses.

\begin{tabular}{lrr}
\hline \hline$M_{\text {ini }} / M_{\odot}$ & $\mathrm{C}$ & $\mathrm{Ba}$ \\
\hline 1.00 & 156 & 2215 \\
1.50 & 49 & 94 \\
1.75 & 25 & 36 \\
1.90 & 26 & 32 \\
2.10 & 14 & 17 \\
2.25 & 15 & 18 \\
2.50 & 12 & 15 \\
2.75 & 9 & 15 \\
3.00 & 10 & 15 \\
3.50 & 12 & 24 \\
4.00 & 18 & 27 \\
5.00 & 89 & 1311 \\
\hline
\end{tabular}

The parallax of $0.0564 \pm 0.0340$ mas is affected by a large error corresponding to relative errors of $60.3 \%$. The object is also contained in the catalog of distances of Bailer-Jones et al. (2018) derived from the DR2 data. They found $9.071_{-1.962}^{+2.939} \mathrm{kpc}$. With our result for the companion mass, we would like to get a clue for the distance for the binary in Hen 2-39. Using the assumed giant $\log g=2.5$, the derived mass of about $2.2 M_{\odot}$ for the Ba star, and the determined $T_{\text {eff }}$, we get

$$
\begin{aligned}
M_{\mathrm{bol}}= & M_{\mathrm{bol}, \odot}+2.5\left(\log g-\log g_{\odot}\right) \\
& -2.5 \log \left(\frac{M}{M_{\odot}}\right)-10 \log \left(\frac{T_{\text {eff }}}{T_{\text {eff } \odot}}\right)=0.262 .
\end{aligned}
$$

The solar values are taken from the Sun Facts Sheet from the NASA Goddard Space Flight Center $^{8}$ and we use $M_{\mathrm{bol}, \odot}=$ $4.74 \mathrm{mag}$ for the Sun derived from the standardized absolute bolometric magnitude scale defined by the international astronomical union (Mamajek et al. 2015). The error of $M_{\text {bol }}$ is

\footnotetext{
8 https://nssdc.gsfc.nasa.gov/planetary/factsheet/ sunfact.html, Version 29-06-2018.
}

dominated by the uncertainty of $\log g$. Assuming an uncertainty of $\Delta \log g=0.5$ and $\Delta M=0.5 M_{\odot}$, we get $\Delta M_{\mathrm{bol}}=1.316$. Nevertheless, we can derive the absolute visual magnitude $M_{\mathrm{V}}=$ $M_{\mathrm{bol}}-\mathrm{BC}(V)=0.852 \pm 1.525$. The bolometric correction of $\mathrm{BC}(V)=-0.590 \pm 0.209$ is calculated using the approach of Alonso et al. (1999) including the preliminary values for $T_{\text {eff }}$ and the $\mathrm{Fe}$ abundance.

We can now estimate the distance via the distance modulus but the known $V$ magnitude shows a much larger uncertainty than the more recent infrared magnitudes (Table 4). By comparing the calculated flux normalized on the $K$ magnitude of Cutri et al. (2003) with the $B$ and $V$ magnitudes of Tylenda et al. (1991) and those for the $I, J$, and $K$ bands from Epchtein et al. (1999), it becomes obvious that our model agrees very well with the brightness values in all filters (Fig. 9). Thus, we decided to rely on the precise $I$ magnitude for the distance estimation. Using the color relation for $(V-I)$ from Alonso et al. (1999) for the given $T_{\text {eff }}$, we find $(V-I)=1.543_{-0.110}^{+0.124} \mathrm{mag}$. This leads to an absolute $M_{I}$ brightness of $M_{I}=-0.691_{-1.649}^{+1.635} \mathrm{mag}$.

Miszalski et al. (2013a) determined an extinction of $E_{\mathrm{B}-\mathrm{V}}=$ 0.37 mag. With the Galactic extinction law with $R_{V}=3.1$ and the relation from Cardelli et al. (1989), we derive the total absorption for the $I$ band of $A_{I}=0.5 A_{V}=0.5 \times 3.1 E_{\mathrm{B}-\mathrm{V}}=0.565 \mathrm{mag}$. Now, we find $d=10^{-\left(M_{I}-I+A_{I}-5\right) / 5}=9.15_{-4.90}^{+10.65} \mathrm{kpc}$. The large error is again an effect of the assumed uncertainty in $\log g$ but, nevertheless, the value agrees with all other distance values within these limits. Furthermore, this value is very close to that derived from the Gaia parallax measurement. With a precise distance measurement, we could get a second handle on the mass of the Ba star. The mass could then be derived by comparing its properties to evolutionary tracks for different masses and compared with our value derived using the abundance yields of evolutionary models.

With its Galactic latitude of $-4.239^{\circ}$ (Gaia Collaboration 2018), the star is located $0.676_{-0.362}^{+0.789} \mathrm{kpc}$ below the Galactic plane, which means that it is just below the edge of the Galactic thin disk (Rix \& Bovy 2013) and, thus, should belong to the thick disk. This assignation is in agreement with the observed low metallicity since simulations for the Galactic metallicity distribution predict negative metallicity gradients for low scale heights and may change the sign at about a scale height of $1.5 \mathrm{kpc}$. Ivezić et al. (2012) also found thick disk stars to be more metal poor (median $[\mathrm{Fe} / \mathrm{H}]=-0.6$ ) compared to thin disk stars (median $[\mathrm{Fe} / \mathrm{H}]=-0.2$ ), where Hen 2-39 lies just in the middle. Furthermore, we can use $M_{\text {bol }}$ to estimate the luminosity $L / L_{\odot}=10^{\left(M_{\text {bol }, \odot}-M_{\text {bol }}\right) / 2.5}=61.83_{-43.43}^{+145.95}$ and the radius

$$
\frac{R}{R_{\odot}}=\sqrt{\frac{L}{L_{\odot}} \frac{T_{\text {eff } \odot}^{4}}{T_{\text {eff }}^{4}}}=13.84_{-6.78}^{+13.38} .
$$

Using the rotational velocity from Sect. 4.1 and the radius the star should have a rotational period of $18.4 \mathrm{~d}$ for a high inclination of $i=90^{\circ}$. Miszalski et al. (2013a) detected a photometrically variability of the star with a period of $5.46 \mathrm{~d}$. Assuming this value for the rotation, we can find an inclination of $i=17.22^{\circ}{ }_{-8.53}^{+18.30}$. The parameters are summarized in Table 5. We speculate that the rotational axis of the giant is perpendicular to the binary orbital plane. The low inclination is then in good agreement with the ring-like appearance of the nebula (Miszalski et al. 2013a), which indicates a nearly pole-on view and therefore a binary orbital plane almost in the plane of the sky (Hillwig et al. 2016). 
L. Löbling et al.: Spectral analysis of the Ba CSPN of Hen 2-39

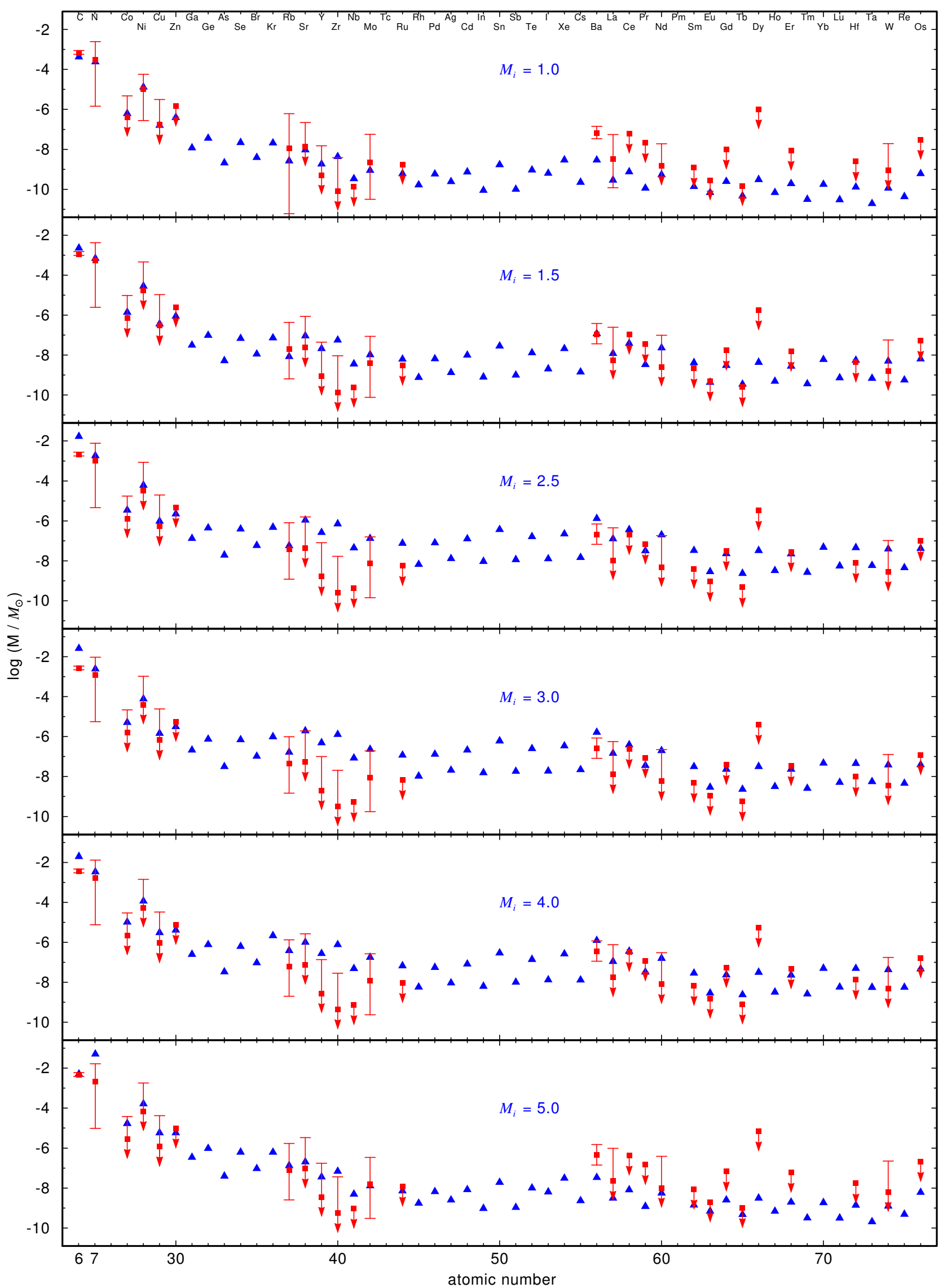

Fig. 8. Total amount of mass ejected during AGB evolution of different elements for evolutionary models from Karakas \& Lugaro (2016) for $Z=0.007$ with initial masses of $1.0-5.0 M_{\odot}$ (blue, initial mass indicated in the panel) compared to the need of accreted mass to reproduce the determined abundance values of the secondary via mass transfer (red). 
Table 4. Brightnesses in different filters for the Ba CSPN of Hen 2-39.

\begin{tabular}{lll}
\hline \hline Filter & Magnitude & Reference \\
\hline$B$ & $17.9 \pm 0.5$ & Tylenda et al. (1991) \\
$V$ & $16.5 \pm 0.5$ & Tylenda et al. (1991) \\
$I$ & $14.68 \pm 0.03$ & Epchtein et al. (1999) \\
$J$ & $13.474 \pm 0.033$ & Cutri et al. (2003) \\
$H$ & $12.614 \pm 0.033$ & Cutri et al. (2003) \\
$K$ & $12.338 \pm 0.030$ & Cutri et al. (2003) \\
\hline
\end{tabular}

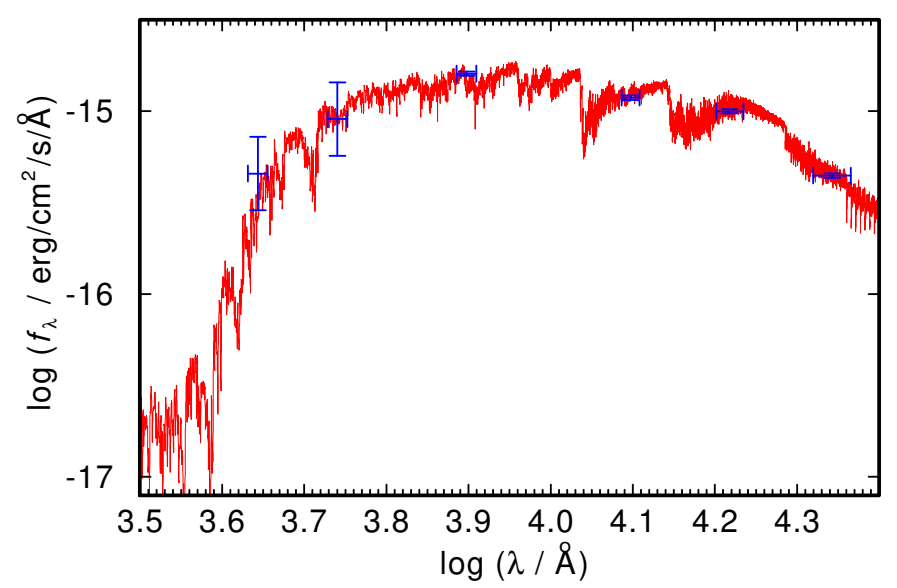

Fig. 9. Synthetic spectrum of our best model of Hen 2-39 normalized to the $H$ magnitude of Cutri et al. (2003) and convolved with a Gaussian with $F W H M=5 \AA$ for clarity, including interstellar reddening with $E_{\mathrm{B}-\mathrm{V}}=0.37$ (red) compared with the observed magnitudes (Table 4).

Table 5. Properties of the CSPN of Hen 2-39.

\begin{tabular}{lc}
\hline \hline$T_{\text {eff }}$ & $(4350 \pm 150) \mathrm{K}$ \\
Distance $^{a}$ & $5.7 \mathrm{kpc}$ \\
Distance $^{b}$ & $\left(9.071_{-1.962}^{+2.939}\right) \mathrm{kpc}$ \\
Distance & $\left(9.15_{-4.90}^{+10.65}\right) \mathrm{kpc}$ \\
$\mathrm{BC}(V)$ & $(-0.590 \pm 0.209) \mathrm{mag}$ \\
$M_{\text {bol }}$ & $0.262 \pm 1.316$ \\
$(V-I)_{0}$ & $1.543_{-0.110}^{+0.124}$ \\
$E(B-V)^{a}$ & -0.37 \\
$L / L_{\odot}$ & $61.83_{-43.43}^{+145.95}$ \\
$M / M_{\odot}$ & $2.2 \pm 0.5$ \\
$\log g$ & $2.5 \pm 0.5$ \\
$R / R_{\odot}$ & $13.84_{-6.78}^{+13.38}$ \\
$\operatorname{Rotation}_{\text {period }}{ }^{a}$ & $5.46 \mathrm{~d}$ \\
$v_{\text {rot }} \sin i$ & $38 \pm 5 \mathrm{~km} \mathrm{~s}^{-1}$ \\
$i$ & $\left(17.22_{-8.53}^{+18.30}\right)$ \\
\hline
\end{tabular}

References. ${ }^{(a)}$ Miszalski et al. (2013a) ${ }^{(b)}$ Bailer-Jones et al. (2018).

\section{Summary and conclusions}

We presented and discussed the spectral analysis of UVES spectra of the Ba CSPN of Hen 2-39. Within the error limits, we confirm the result of Miszalski et al. (2013a) that the observed nucleus of Hen 2-39 has a cool atmosphere of $T_{\text {eff }}=(4350 \pm$ 150) K. Furthermore, we confirm the $\mathrm{C}$ and $\mathrm{Ba}$ enrichment but can significantly improve the abundances of $[\mathrm{C} / \mathrm{H}]=0.36 \pm 0.08$ and $[\mathrm{Ba} / \mathrm{Fe}]=1.8 \pm 0.50$ due to the high resolution of the analyzed spectra. We determined abundances or upper abundance limits for 26 trans-iron elements for the first time. For Tc, the lightest element with no stable isotope, we find an upper abundance limit of $\log \epsilon_{\mathrm{Tc}}<2.5$. This does not confirm the presence of $\mathrm{Tc}$ in the atmosphere of the star proving prior mass transfer. The limiting factor is not the resolution of the spectrum. For a clear detection of Tc, an exposure time about six times longer than that of all the spectra combined would be required to obtain the necessary $\mathrm{S} / \mathrm{N}$ ratio. We can find a low metallicity of $[\mathrm{Fe} / \mathrm{H}]=-0.3 \pm 1.0$ for the $\mathrm{Ba}$ giant. The determined abundance pattern requires mass transfer from a companion with an extremely high enrichment of AGB nucleosynthesis products. The comparison with nucleosynthesis models of Karakas \& Lugaro (2016) indicates an initial mass of 1.75-3.00 $M_{\odot}$ for the primary. The percentage of ejected mass that needs to be accreted indicates that the preferred mass transfer mechanism is wind-RLOF. A wide binary involving Bondi-Hoyle accretion can be ruled out as can a CE evolution. For this star, the distance is rather uncertain. Thus, it cannot be used for a spectroscopic determination of the mass by interpolation from evolutionary tracks. A precise spectroscopic determination of the distance is hampered by the fact that $\log g$ cannot be constrained within narrow error limits from the analysis of the spectra. It is highly desirable to get a more precise distance measurement. This would also help to get a second value for the mass of the $\mathrm{Ba}$ CSPN to compare with that derived from the comparison with AGB models. With this second measurement, it would be possible to refine the primary mass estimate and place stronger constraints on the mass transfer. Our result for the height above the Galactic plane places this system among the thick disk population, in good agreement with the subsolar metallicity derived by our analysis.

Acknowledgements. We thank the anonymous referee for their constructive review of the manuscript. We thank Brent Miszalski and Thomas Rauch for their helpful comments and suggestions. L.L. is supported by the German Research Foundation (DFG, grant WE 1312/49-1) and by the Studentship Programme of the European Southern Observatory. D.J. gratefully acknowledges the Spanish Ministry of Economy and Competitiveness (MINECO) under the grant AYA2017-83383-P. This research has made use of NASA's Astrophysics Data System and the SIMBAD database, operated at CDS, Strasbourg, France. This work has made use of data from the European Space Agency (ESA) mission Gaia (https://www . cosmos.esa.int/gaia), processed by the Gaia Data Processing and Analysis Consortium (DPAC, https://www. cosmos.esa.int/web/ gaia/dpac/consortium). Funding for the DPAC has been provided by national institutions, in particular the institutions participating in the Gaia Multilateral Agreement.

\section{References}

Abate, C., Pols, O. R., Izzard, R. G., Mohamed, S. S., \& de Mink, S. E. 2013, A\&A, 552, A26

Acker, A., Marcout, J., Ochsenbein, F., et al. 1992, The Strasbourg-ESO Catalogue of Galactic Planetary Nebulae. Parts I, II (Garching: European Southern Observatory)

Aller, A., Lillo-Box, J., Vučković, M., et al. 2018, MNRAS, 476, 1140

Alonso, A., Arribas, S., \& Martínez-Roger, C. 1999, A\&AS, 140, 261

Asplund, M., Grevesse, N., Sauval, A. J., \& Scott, P. 2009, ARA\&A, 47, 481

Bailer-Jones, C. A. L., Rybizki, J., Fouesneau, M., Mantelet, G., \& Andrae, R. 2018, AJ, 156, 58

Bidelman, W. P., \& Keenan, P. C. 1951, ApJ, 114, 473

Blanco-Cuaresma, S., Soubiran, C., Heiter, U., \& Jofré, P. 2014, A\&A, 569, A111

Boffin, H. M. J. 2015, Ecology of Blue Straggler Stars (Berlin, Heidelberg: Springer-Verlag)

Boffin, H. M. J., \& Jorissen, A. 1988, A\&A, 205, 155

Bond, H. E., Pollacco, D. L., \& Webbink, R. F. 2003, AJ, 125, 260

Buckley, D. A. H., Swart, G. P., \& Meiring, J. G. 2006, Proc. SPIE, 6267, 62670 Z 
Burgh, E. B., Nordsieck, K. H., Kobulnicky, H. A., et al. 2003, in Instrument Design and Performance for Optical/Infrared Ground-based Telescopes, eds. M. Iye, \& A. F. M. Moorwood, Proc. SPIE, 4841, 1463

Cardelli, J. A., Clayton, G. C., \& Mathis, J. S. 1989, ApJ, 345, 245

Castelli, F., \& Kurucz, R. L. 2003, in Modelling of Stellar Atmospheres, eds. N. Piskunov, W. W. Weiss, \& D. F. Gray, IAU Symp., 210, A20

Chen, Z., Frank, A., Blackman, E. G., Nordhaus, J., \& Carroll-Nellenback, J. 2017, MNRAS, 468, 4465

Corradi, R. L. M., Sabin, L., Miszalski, B., et al. 2011, MNRAS, 410, 1349

Cutri, R. M., Skrutskie, M. F., van Dyk, S., et al. 2003, VizieR Online Data Catalog: II/246

de Castro, D. B., Pereira, C. B., Roig, F., et al. 2016, MNRAS, 459, 4299

De Marco O. 2009, PASP, 121, 316

Dekker, H., D’Odorico, S., Kaufer, A., Delabre, B., \& Kotzlowski, H. 2000, in Optical and IR Telescope Instrumentation and Detectors, eds. M. Iye, \& A. F. Moorwood, Proc. SPIE, 4008, 534

Epchtein, N., Deul, E., Derriere, S., et al. 1999, VizieR Online Data Catalog: II $/ 240$

Frew, D. J., Parker, Q. A., \& Bojičić, I. S. 2016, MNRAS, 455, 1459

Gaia Collaboration 2018, VizieR Online Data Catalog: I/345

Gray, R. O., \& Corbally, C. J. 1994, AJ, 107, 742

Gray, R. O., McGahee, C. E., Griffin, R. E. M., \& Corbally, C. J. 2011, AJ, 141, 160

Grevesse, N., \& Sauval, A. J. 1998, Space Sci. Rev., 85, 161

Han, Z., Eggleton, P. P., Podsiadlowski, P., \& Tout, C. A. 1995, MNRAS, 277, 1443

Henize, K. G. 1967, ApJS, 14, 125

Herwig, F. 2005, ARA\&A, 43, 435

Hillwig, T. C., Jones, D., De Marco, O., et al. 2016, ApJ, 832, 125

Hubeny, I., Mihalas, D., \& Werner, K., eds. 2003, in Stellar Atmosphere Modeling, ASP Conf. Ser., 288, 17

Husti, L., Gallino, R., Bisterzo, S., Straniero, O., \& Cristallo, S. 2009, PASA, 26 , 176

Iben, Jr. I., \& Livio, M. 1993, PASP, 105, 1373

Ivezić, Ž., Beers, T. C., \& Jurić, M. 2012, ARA\&A, 50, 251

Jones, D., \& Boffin, H. M. J. 2017, Nat. Astron., 1, 0117

Jones, D., Van Winckel, H., Aller, A., Exter, K., \& De Marco, O. 2017, A\&A, 600, L9

Jorissen, A., \& Mayor, M. 1988, A\&A, 198, 187

Jorissen, A., Van Eck, S., Mayor, M., \& Udry, S. 1998, A\&A, 332, 877

Joss, P. C., Rappaport, S., \& Lewis, W. 1987, ApJ, 319, 180

Karakas, A. I., \& Lattanzio, J. C. 2014, PASA, 31, e030

Karakas, A. I., \& Lugaro, M. 2016, ApJ, 825, 26

Karakas, A. I., Lugaro, M., Carlos, M., et al. 2018, MNRAS, 477, 421

Karinkuzhi, D., Goswami, A., Sridhar, N., Masseron, T., \& Purandardas, M. 2018, MNRAS, 476, 3086
Kobulnicky, H. A., Nordsieck, K. H., Burgh, E. B., et al. 2003, in Instrument Design and Performance for Optical/Infrared Ground-based, eds. M. Iye \& A. F. M. Moorwood Telescopes, Proc. SPIE, 4841, 1634

Kurucz, R. L. 1991, in Stellar Atmospheres: Beyond Classical Models, eds L. Crivellari, I. Hubeny, \& D. G. Hummer (Berlin: Springer Science \& Business Media), NATO ASIC Proc., 341, 441

Lebzelter, T., \& Hron, J. 2003, A\&A, 411, 533

Madonna, S., García-Rojas, J., Sterling, N. C., et al. 2017, MNRAS, 471, 1341

Madonna, S., Bautista, M. A., Dinerstein, H., et al. 2018, ApJL, 861, L8

Mamajek, E. E., Torres, G., Prsa, A., et al. 2015, ArXiv e-prints [arXiv:1510.06262]

Masseron, T., Merle, T., \& Hawkins, K. 2016, Astrophysics Source Code Library [record ascl:1605.004]

McClure, R. D. 1983, ApJ, 268, 264

McClure, R. D., Fletcher, J. M., \& Nemec, J. M. 1980, ApJ, 238, L35

McClure, R. D., \& Woodsworth, A. W. 1990, ApJ, 352, 709

Merle, T., Jorissen, A., Van Eck, S., Masseron, T., \& Van Winckel, H. 2016, A\&A, 586, A151

Merrill, P. W. 1952, ApJ, 116, 21

Miller Bertolami, M. M. 2016, A\&A, 588, A25

Miszalski, B., Boffin, H. M. J., Frew, D. J., et al. 2012, MNRAS, 419, 39

Miszalski, B., Boffin, H. M. J., Jones, D., et al. 2013a, MNRAS, 436, 3068

Miszalski, B., Boffin, H. M. J., \& Corradi, R. L. M. 2013b, MNRAS, 428, L39

Mohamed, S., \& Podsiadlowski, P. 2007, in 15th European Workshop on White Dwarfs, eds. R. Napiwotzki \& M. R. Burleigh, ASP Conf. Ser., 372, 397

Nagae, T., Oka, K., Matsuda, T., et al. 2004, A\&A, 419, 335

Neyskens, P., van Eck, S., Jorissen, A., et al. 2015, Nature, 517, 174

Palmeri, P., Quinet, P., Biémont, É., et al. 2007, MNRAS, 374, 63

Rix, H.-W., \& Bovy, J. 2013, A\&ARv, 21, 61

Saladino, M. I., Pols, O. R., van der Helm, E., Pelupessy, I., \& Portegies Zwart, S. 2018, A\&A, 618, A50

Smiljanic, R., Barbuy, B., de Medeiros, J. R., \& Maeder, A. 2006, Rev. Mex. Astron. Astrofis. Conf. Ser., 26, 45

Sneden, C., Lucatello, S., Ram, R. S., Brooke, J. S. A., \& Bernath, P. 2014, ApJS, 214, 26

Tabernero, H. M., Dorda, R., Negueruela, I., \& González-Fernández, C. 2018 MNRAS, 476, 3106

Theuns, T., Boffin, H. M. J., \& Jorissen, A. 1996, MNRAS, 280, 1264

Thevenin, F., \& Jasniewicz, G. 1997, A\&A, 320, 913

Tylenda, R., Acker, A., Stenholm, B., Gleizes, F., \& Raytchev, B. 1991, A\&AS, 89,77

Tyndall, A. A., Jones, D., Boffin, H. M. J., et al. 2013, MNRAS, 436, 2082

Van Eck, S., \& Jorissen, A. 1999, A\&A, 345, 127

Werner, K., \& Herwig, F. 2006, PASP, 118, 183

Wray, J. D. 1966, Ph.D. Thesis, Northwestern University, IL, USA 
Appendix A: Additional figures and tables

Table A.1. Observation log for the UVES observations.

\begin{tabular}{|c|c|c|c|}
\hline Start time (UT) & $\lambda(\AA)$ & Exp. time (s) & $S / N$ \\
\hline 2014-04-03 02:42:47 & $5654-9465$ & 1500 & 14.8 \\
\hline 2014-04-03 02:42:51 & $3732-5000$ & 1500 & 3.7 \\
\hline 2014-04-03 03:08:39 & $5654-9465$ & 1500 & 14.1 \\
\hline 2014-04-03 03:08:39 & $3732-5000$ & 1500 & 3.3 \\
\hline 2014-04-03 03:36:22 & $5654-9465$ & 1500 & 12.6 \\
\hline 2014-04-03 03:36:26 & $3732-5000$ & 1500 & 3.1 \\
\hline 2014-04-03 04:02:13 & $5654-9465$ & 1500 & 14.5 \\
\hline 2014-04-03 04:02:14 & $3732-5000$ & 1500 & 3.7 \\
\hline 2014-04-04 00:40:42 & $5654-9465$ & 1500 & 13.5 \\
\hline 2014-04-04 00:40:46 & $3732-5000$ & 1500 & 3.1 \\
\hline 2014-04-04 01:06:34 & $5654-9465$ & 1500 & 13.1 \\
\hline 2014-04-04 01:06:34 & $3732-5000$ & 1500 & 2.9 \\
\hline 2014-04-04 01:34:52 & $5654-9465$ & 1500 & 14.8 \\
\hline 2014-04-04 01:34:59 & $3732-5000$ & 1500 & 3.7 \\
\hline 2014-04-04 02:00:47 & $3732-5000$ & 1500 & 3.5 \\
\hline 2014-04-04 02:00:47 & $5654-9465$ & 1500 & 14.8 \\
\hline 2014-04-04 02:32:05 & $5654-9465$ & 1500 & 14.5 \\
\hline 2014-04-04 02:32:11 & $3732-5000$ & 1500 & 3.2 \\
\hline 2014-04-04 02:57:59 & $3732-5000$ & 1500 & 3.3 \\
\hline 2014-04-04 02:58:00 & $5654-9465$ & 1500 & 14.1 \\
\hline 2014-04-13 02:30:36 & $5654-9465$ & 1450 & 12.9 \\
\hline 2014-04-13 02:30:40 & $3732-5000$ & 1450 & 2.9 \\
\hline 2014-04-13 02:55:38 & $3732-5000$ & 1450 & 3.2 \\
\hline 2014-04-13 02:55:39 & $5654-9465$ & 1450 & 13.7 \\
\hline 2014-04-15 00:20:10 & $5654-9465$ & 1450 & 14.3 \\
\hline 2014-04-15 00:20:14 & $3732-5000$ & 1450 & 3.0 \\
\hline 2014-04-15 00:45:11 & $5654-9465$ & 1450 & 11.3 \\
\hline 2014-04-15 00:45:12 & $3732-5000$ & 1450 & 2.4 \\
\hline 2014-04-15 01:10:37 & $5654-9465$ & 1450 & 12.9 \\
\hline 2014-04-15 01:10:41 & $3732-5000$ & 1450 & 2.7 \\
\hline 2014-04-15 01:35:39 & $3732-5000$ & 1450 & 2.9 \\
\hline 2014-04-15 01:35:39 & $5654-9465$ & 1450 & 13.4 \\
\hline 2014-04-15 02:01:20 & $5654-9465$ & 1500 & 13.1 \\
\hline 2014-04-15 02:01:24 & $3732-5000$ & 1500 & 2.8 \\
\hline 2014-04-15 02:27:11 & $5654-9465$ & 1500 & 13.1 \\
\hline 2014-04-15 02:27:12 & $3732-5000$ & 1500 & 2.6 \\
\hline
\end{tabular}


Table A.2. Diagnostic lines used for the determination of stellar parameters.

\begin{tabular}{|c|c|c|c|c|c|}
\hline$\lambda$ interval $(\AA)$ & $\lambda_{\text {line }}(\AA)$ & Ion & $E_{\text {low }}\left(\mathrm{cm}^{-1}\right)$ & $E_{\text {up }}\left(\mathrm{cm}^{-1}\right)$ & $\log g f$ \\
\hline $4020.400 \pm 1.30$ & 4020.400 & Sc I & 0 & 24866 & -0.130 \\
\hline $4023.690 \pm 1.30$ & 4023.690 & Sc I & 168 & 25014 & 0.210 \\
\hline $4045.820 \pm 1.30$ & 4045.820 & $\mathrm{Fe} \mathrm{I}$ & 11976 & 36686 & 0.280 \\
\hline $4054.544 \pm 1.30$ & 4054.544 & Sc I & 0 & 24657 & -0.750 \\
\hline $4063.605 \pm 1.30$ & 4063.605 & $\mathrm{Fe} I$ & 12561 & 37163 & 0.072 \\
\hline $4071.740 \pm 1.30$ & 4071.740 & $\mathrm{Fe} \mathrm{I}$ & 12969 & 37521 & -0.022 \\
\hline $4082.390 \pm 1.30$ & 4082.390 & Sc I & 168 & 24657 & -0.444 \\
\hline $4233.170 \pm 1.30$ & 4233.170 & Fe II & 20831 & 44447 & -1.995 \\
\hline $4271.760 \pm 1.30$ & 4271.760 & $\mathrm{Fe} I$ & 11976 & 35379 & -0.164 \\
\hline \multirow[t]{2}{*}{$4305.820 \pm 1.40$} & 4305.720 & Sc II & 4803 & 28021 & -1.200 \\
\hline & 4305.910 & Ti I & 6843 & 30060 & 0.300 \\
\hline $4307.900 \pm 1.30$ & 4307.900 & $\mathrm{Fe} I$ & 12561 & 35768 & -0.300 \\
\hline $4314.080 \pm 1.30$ & 4314.080 & Sc II & 4988 & 28161 & -0.220 \\
\hline $4320.750 \pm 1.30$ & 4320.750 & Sc II & 4883 & 28021 & -0.100 \\
\hline $4325.010 \pm 1.30$ & 4325.010 & Sc II & 4803 & 27918 & -0.250 \\
\hline $4325.760 \pm 1.30$ & 4325.760 & $\mathrm{Fe} I$ & 12969 & 36079 & -0.300 \\
\hline $4351.769 \pm 1.30$ & 4351.769 & Fe II & 21812 & 44785 & -2.100 \\
\hline $4374.472 \pm 1.30$ & 4374.472 & Sc II & 4988 & 27841 & -0.640 \\
\hline $4383.550 \pm 1.30$ & 4383.550 & $\mathrm{Fe}_{\mathrm{I}}$ & 11976 & 34782 & 0.200 \\
\hline $4395.040 \pm 1.30$ & 4395.040 & Ti II & 8744 & 31491 & -0.660 \\
\hline $4400.398 \pm 1.30$ & 4400.398 & Sc II & 4883 & 27602 & -0.480 \\
\hline $4404.761 \pm 1.30$ & 4404.761 & $\mathrm{Fe} \mathrm{I}$ & 12561 & 35257 & -0.142 \\
\hline $4415.560 \pm 1.30$ & 4415.560 & Sc II & 4803 & 27444 & -0.510 \\
\hline $4443.812 \pm 1.30$ & 4443.812 & Ti II & 8710 & 31207 & -0.690 \\
\hline $4468.500 \pm 1.30$ & 4468.500 & Ti II & 9118 & 31491 & -0.270 \\
\hline $4501.273 \pm 1.30$ & 4501.273 & Ti II & 8998 & 31207 & -0.684 \\
\hline $4522.634 \pm 1.30$ & 4522.634 & Fe II & 22939 & 45044 & -2.119 \\
\hline \multirow[t]{4}{*}{$4534.400 \pm 2.50$} & 4533.239 & Ti I & 6843 & 28896 & 0.563 \\
\hline & 4533.969 & Ti II & 9976 & 32025 & -0.612 \\
\hline & 4534.778 & Ti I & 6743 & 28788 & 0.376 \\
\hline & 4535.570 & Ti I & 6661 & 28703 & 0.172 \\
\hline \multirow[t]{2}{*}{$4549.550 \pm 1.45$} & 4549.474 & Fe II & 22810 & 44785 & -1.957 \\
\hline & 4549.617 & Ti II & 12775 & 34748 & -0.110 \\
\hline $4555.893 \pm 1.30$ & 4555.893 & Fe II & 22810 & 44754 & -2.281 \\
\hline $4563.761 \pm 1.30$ & 4563.761 & Ti II & 9851 & 31757 & -0.795 \\
\hline $4571.968 \pm 1.30$ & 4571.968 & Ti II & 12677 & 34543 & -0.209 \\
\hline $4629.339 \pm 1.30$ & 4629.339 & $\mathrm{Fe}$ II & 22637 & 44233 & -2.379 \\
\hline $4670.407 \pm 1.30$ & 4670.407 & Sc II & 10945 & 32350 & -0.518 \\
\hline \multirow[t]{8}{*}{$4920.000 \pm 5.00$} & 4915.233 & Ti I & 15220 & 35560 & -0.945 \\
\hline & 4918.954 & $\mathrm{Fe} \mathrm{I}$ & 33507 & 53831 & -0.672 \\
\hline & 4918.993 & $\mathrm{Fe} \mathrm{I}$ & 23111 & 43435 & -0.365 \\
\hline & 4919.867 & Ti I & 17424 & 37744 & -0.260 \\
\hline & 4920.502 & $\mathrm{Fe} \mathrm{I}$ & 22846 & 43163 & 0.058 \\
\hline & 4921.769 & Ti I & 17540 & 37852 & -0.005 \\
\hline & 4922.827 & Sc I & 16023 & 36331 & -0.418 \\
\hline & 4923.927 & $\mathrm{Fe}$ II & 23318 & 43621 & -1.319 \\
\hline $4981.732 \pm 1.30$ & 4981.732 & Ti I & 6843 & 26911 & 0.586 \\
\hline $5701.545 \pm 1.00$ & 5701.545 & $\mathrm{Fe} I$ & 20641 & 38175 & -1.565 \\
\hline $5705.464 \pm 1.00$ & 5705.466 & $\mathrm{Fe} \mathrm{I}$ & 34692 & 52214 & -1.581 \\
\hline \multirow[t]{2}{*}{$5732.000 \pm 1.30$} & 5731.762 & $\mathrm{Fe} \mathrm{I}$ & 34329 & 51771 & -1.174 \\
\hline & 5732.275 & Fe I & 40257 & 57698 & -1.191 \\
\hline $5747.954 \pm 1.00$ & 5747.954 & $\mathrm{Fe} \mathrm{I}$ & 37163 & 54555 & -0.599 \\
\hline $5861.108 \pm 1.00$ & 5861.107 & $\mathrm{Fe} I$ & 34547 & 51604 & -2.761 \\
\hline $5934.654 \pm 1.00$ & 5934.653 & $\mathrm{Fe} \mathrm{I}$ & 31686 & 48532 & -1.192 \\
\hline \multirow[t]{2}{*}{$5952.800 \pm 1.00$} & 5952.716 & $\mathrm{Fe} I$ & 32134 & 48928 & -2.513 \\
\hline & 5952.889 & $\mathrm{Fe} \mathrm{I}$ & 34040 & 50833 & -3.725 \\
\hline $6151.617 \pm 1.00$ & 6151.617 & $\mathrm{Fe} I$ & 17550 & 33802 & -3.582 \\
\hline $6165.360 \pm 1.00$ & 6165.361 & $\mathrm{Fe} I$ & 33413 & 49628 & -1.667 \\
\hline
\end{tabular}

Notes. If no interval is given, the line belongs to the previous interval. 
Table A.2. continued.

\begin{tabular}{|c|c|c|c|c|c|}
\hline$\lambda$ interval $(\AA)$ & $\lambda_{\text {line }}(\AA)$ & Ion & $E_{\text {low }}\left(\mathrm{cm}^{-1}\right)$ & $E_{\text {up }}\left(\mathrm{cm}^{-1}\right)$ & $\log g f$ \\
\hline \multirow[t]{2}{*}{$6170.500 \pm 1.00$} & 6170.504 & $\mathrm{Fe} I$ & 38678 & 54880 & -0.654 \\
\hline & 6171.006 & $\mathrm{Fe} \mathrm{I}$ & 38175 & 54376 & -1.788 \\
\hline \multirow[t]{2}{*}{$6173.334 \pm 1.00$} & 6173.341 & $\mathrm{Fe} I$ & 17927 & 34122 & -3.081 \\
\hline & 6173.642 & $\mathrm{Fe} \mathrm{I}$ & 35856 & 52050 & -3.413 \\
\hline $6191.500 \pm 1.00$ & 6191.558 & $\mathrm{Fe} \mathrm{I}$ & 19621 & 35768 & -1.287 \\
\hline $6210.658 \pm 1.30$ & 6210.658 & Sc I & 0 & 16097 & -1.090 \\
\hline $6265.132 \pm 1.00$ & 6265.141 & $\mathrm{Fe} \mathrm{I}$ & 17550 & 33507 & -2.834 \\
\hline $6305.657 \pm 1.30$ & 6305.657 & Sc I & 168 & 16023 & -0.950 \\
\hline $6318.000 \pm 1.00$ & 6318.018 & $\mathrm{Fe} I$ & 19788 & 35612 & -2.338 \\
\hline $6336.823 \pm 1.00$ & 6336.830 & $\mathrm{Fe} I$ & 29733 & 45509 & -1.260 \\
\hline \multirow{3}{*}{$6408.000 \pm 1.00$} & 6407.643 & $\mathrm{Fe} \mathrm{I}$ & 32874 & 48476 & -3.620 \\
\hline & 6408.026 & $\mathrm{Fe} \mathrm{I}$ & 29733 & 45334 & -1.230 \\
\hline & 6408.332 & $\mathrm{Fe} \mathrm{I}$ & 35379 & 50980 & -3.563 \\
\hline $6475.624 \pm 1.00$ & 6475.632 & $\mathrm{Fe} \mathrm{I}$ & 20641 & 36079 & -3.070 \\
\hline $6481.870 \pm 1.00$ & 6481.878 & $\mathrm{Fe} I$ & 18378 & 33802 & -3.080 \\
\hline \multirow[t]{3}{*}{$6807.000 \pm 1.00$} & 6806.622 & $\mathrm{Fe} \mathrm{I}$ & 44023 & 58710 & -1.744 \\
\hline & 6806.843 & $\mathrm{Fe} I$ & 21999 & 36686 & -3.210 \\
\hline & 6807.288 & $\mathrm{Fe} I$ & 42533 & 57219 & -2.735 \\
\hline $6810.262 \pm 1.00$ & 6810.262 & $\mathrm{Fe} \mathrm{I}$ & 37158 & 51837 & -1.120 \\
\hline $8434.957 \pm 1.30$ & 8434.957 & Ti I & 6843 & 18695 & -0.886 \\
\hline \multirow[t]{2}{*}{$8514.400 \pm 1.90$} & 8514.072 & $\mathrm{Fe} \mathrm{I}$ & 17727 & 29469 & -2.229 \\
\hline & 8515.109 & $\mathrm{Fe} I$ & 24339 & 36079 & -2.073 \\
\hline \multirow[t]{2}{*}{$8518.300 \pm 1.50$} & 8518.028 & Ti I & 17215 & 28952 & -1.250 \\
\hline & 8518.352 & Ti I & 15157 & 26893 & -1.089 \\
\hline $8582.350 \pm 1.35$ & 8582.258 & $\mathrm{Fe} \mathrm{I}$ & 24119 & 35768 & -2.133 \\
\hline $8611.800 \pm 0.90$ & 8611.803 & $\mathrm{Fe} I$ & 22947 & 34556 & -1.900 \\
\hline \multirow[t]{2}{*}{$8679.000 \pm 2.10$} & 8678.997 & $\mathrm{Fe} \mathrm{I}$ & 48516 & 60035 & -3.806 \\
\hline & 8679.632 & $\mathrm{Fe} \mathrm{I}$ & 40052 & 51570 & -1.512 \\
\hline $8682.900 \pm 1.30$ & 8682.979 & Ti I & 8492 & 20006 & -1.941 \\
\hline $8688.950 \pm 1.65$ & 8688.624 & $\mathrm{Fe} \mathrm{I}$ & 17550 & 29056 & -1.212 \\
\hline $8692.000 \pm 1.00$ & 8692.331 & Ti I & 8437 & 19938 & -2.295 \\
\hline \multirow[t]{4}{*}{$8711.500 \pm 3.00$} & 8710.174 & Mg I & 47841 & 59319 & -1.550 \\
\hline & 8710.392 & $\mathrm{Fe} I$ & 39626 & 51103 & -0.555 \\
\hline & 8712.676 & Mg I & 47844 & 59319 & -1.670 \\
\hline & 8713.188 & $\mathrm{Fe} I$ & 23784 & 35257 & -3.148 \\
\hline $8730.750 \pm 0.95$ & 8730.497 & Ti I & 27026 & 38477 & -2.024 \\
\hline \multirow[t]{2}{*}{$8735.250 \pm 1.75$} & 8734.712 & Ti I & 8492 & 19938 & -2.384 \\
\hline & 8736.020 & Mg I & 47957 & 59401 & -0.690 \\
\hline $8742.250 \pm 0.75$ & 8742.446 & Si I & 47352 & 58787 & -0.630 \\
\hline $8757.200 \pm 1.60$ & 8757.187 & $\mathrm{Fe} I$ & 22947 & 34363 & -2.026 \\
\hline $8792.850 \pm 1.35$ & 8793.342 & $\mathrm{Fe} \mathrm{I}$ & 37163 & 48532 & -0.196 \\
\hline \multirow[t]{2}{*}{$8806.000 \pm 2.70$} & 8806.756 & Mg I & 35051 & 46403 & -0.137 \\
\hline & 8808.170 & $\mathrm{Fe} \mathrm{I}$ & 40405 & 51754 & -1.109 \\
\hline $8824.360 \pm 1.15$ & 8824.220 & $\mathrm{Fe} \mathrm{I}$ & 17727 & 29056 & -1.540 \\
\hline $8838.750 \pm 1.25$ & 8838.428 & $\mathrm{Fe} \mathrm{I}$ & 23052 & 34363 & -1.980 \\
\hline
\end{tabular}




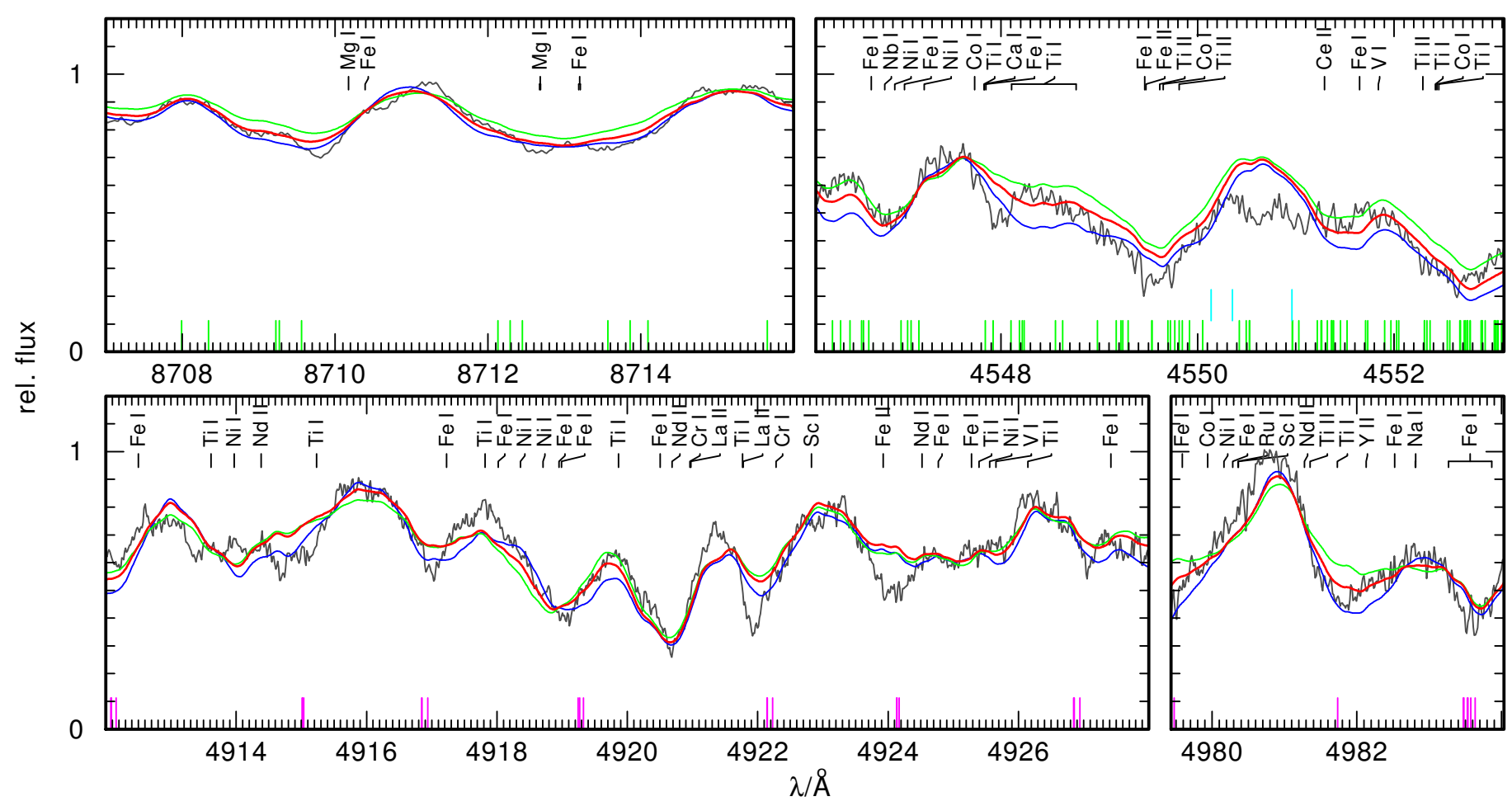

Fig. A.1. Observation (gray) of Hen 2-39 compared to model spectra with $T_{\text {eff }}=4000,4250,4500 \mathrm{~K}$ (blue, red, green) for selected regions that were used for the determination of $T_{\text {eff }} . \mathrm{CN}, \mathrm{C}_{2}$, and $\mathrm{CH}$ absorption lines are indicated at the bottom in green, purple, and cyan, respectively. All absorption lines that appear with an equivalent width $\geq 20 \mathrm{~m} \AA$ in the calculated spectrum are indicated.



Fig. A.2. Like Fig. A.1, for $\log g=2.0,2.5,3.0$ (blue, red, green). 
A\&A 624, A1 (2019)

Table A.3. Diagnostic lines used for our determination of element abundances.

\begin{tabular}{|c|c|c|c|c|}
\hline$\lambda_{\text {line }}(\AA)$ & Ion & $E_{\text {low }}\left(\mathrm{cm}^{-1}\right)$ & $E_{\text {up }}\left(\mathrm{cm}^{-1}\right)$ & $\log g f$ \\
\hline 5889.951 & $\mathrm{Na} I$ & 0 & 16973 & 0.101 \\
\hline 5895.924 & $\mathrm{Na} \mathrm{I}$ & 0 & 16956 & -0.197 \\
\hline 6696.185 & $\mathrm{Al} \mathrm{I}$ & 32435 & 47365 & -1.576 \\
\hline 6698.670 & $\mathrm{Al} \mathrm{I}$ & 25438 & 40272 & -1.960 \\
\hline 6698.673 & $\mathrm{Al} \mathrm{I}$ & 25348 & 40272 & -1.647 \\
\hline 6905.646 & $\mathrm{Al} \mathrm{I}$ & 32435 & 46912 & -1.287 \\
\hline 7083.969 & $\mathrm{Al} \mathrm{I}$ & 32435 & 46548 & -1.111 \\
\hline 7361.568 & $\mathrm{Al} \mathrm{I}$ & 32435 & 46016 & -0.903 \\
\hline 7835.309 & Al I & 32435 & 45195 & -0.649 \\
\hline 7836.134 & Al I & 32437 & 45195 & -0.494 \\
\hline 7836.134 & $\mathrm{Al} \mathrm{I}$ & 32437 & 45195 & -1.795 \\
\hline 8773.896 & $\mathrm{Al} \mathrm{I}$ & 32437 & 43831 & -0.161 \\
\hline 8773.898 & $\mathrm{Al} \mathrm{I}$ & 32437 & 43831 & -1.462 \\
\hline 7725.046 & $\mathrm{~S}_{\mathrm{I}}$ & 9239 & 22180 & -6.000 \\
\hline 7698.974 & $\mathrm{~K}_{\mathrm{I}}$ & 0 & 12985 & -0.170 \\
\hline 5857.451 & $\mathrm{Ca} \mathrm{I}$ & 23652 & 40720 & 0.257 \\
\hline 6161.297 & $\mathrm{Ca}$ I & 20349 & 36575 & -1.293 \\
\hline 6162.173 & $\mathrm{Ca} \mathrm{I}$ & 15316 & 31539 & -0.167 \\
\hline 6169.042 & Ca I & 20349 & 36555 & -0.804 \\
\hline 6169.563 & $\mathrm{Ca} I$ & 20371 & 36575 & -0.527 \\
\hline 6343.308 & $\mathrm{Ca}$ I & 35819 & 51579 & 0.845 \\
\hline 6361.786 & $\mathrm{Ca} \mathrm{I}$ & 35897 & 51611 & 0.954 \\
\hline 6449.810 & $\mathrm{Ca} I$ & 20335 & 35835 & -0.550 \\
\hline 6455.600 & $\mathrm{Ca} \mathrm{I}$ & 20349 & 35835 & -1.350 \\
\hline 6462.570 & $\mathrm{Ca}$ I & 20349 & 35819 & 0.310 \\
\hline 6471.668 & $\mathrm{Ca} \mathrm{I}$ & 20371 & 35819 & -0.680 \\
\hline 6493.788 & $\mathrm{Ca} \mathrm{I}$ & 20335 & 35730 & 0.140 \\
\hline 6499.654 & $\mathrm{Ca} \mathrm{I}$ & 20349 & 35730 & -0.650 \\
\hline 8498.023 & Ca II & 13650 & 25414 & -1.312 \\
\hline 8542.091 & $\mathrm{Ca}$ II & 13711 & 25414 & -0.362 \\
\hline 8662.141 & Ca II & 13650 & 25192 & -0.623 \\
\hline 4729.200 & Sc I & 11520 & 32659 & -0.502 \\
\hline 4729.236 & Sc I & 11558 & 32697 & -0.385 \\
\hline 4734.105 & Sc I & 11520 & 32637 & -0.110 \\
\hline 4753.161 & Sc I & 0 & 21033 & -1.658 \\
\hline 4779.348 & Sc I & 168 & 21086 & -1.613 \\
\hline 4791.511 & Sc I & 168 & 21033 & -2.075 \\
\hline 5700.164 & Sc I & 11558 & 29096 & 0.290 \\
\hline 5717.307 & Sc I & 11610 & 29096 & -0.505 \\
\hline 5724.107 & Sc I & 11558 & 29023 & -0.627 \\
\hline 6305.657 & Sc I & 168 & 16023 & -0.950 \\
\hline 6378.807 & Sc I & 0 & 15673 & -2.632 \\
\hline 6413.324 & Sc I & 168 & 15757 & -2.677 \\
\hline 4314.080 & Sc II & 4988 & 28161 & -0.220 \\
\hline 4431.370 & Sc II & 4883 & 27444 & -1.830 \\
\hline 6279.740 & Sc II & 12102 & 28021 & -1.265 \\
\hline 6309.920 & Sc II & 12074 & 27918 & -1.630 \\
\hline 4455.320 & Ti I & 11640 & 34079 & 0.480 \\
\hline 4518.023 & $\mathrm{Ti} \mathrm{I}$ & 6661 & 28788 & -0.252 \\
\hline 4522.796 & Ti I & 6599 & 28703 & -0.265 \\
\hline 4533.239 & Ti I & 6843 & 28896 & 0.563 \\
\hline 4534.778 & Ti I & 6743 & 28788 & 0.376 \\
\hline 4535.570 & Ti I & 6661 & 28703 & 0.172 \\
\hline 4535.916 & $\mathrm{Ti}$ I & 6599 & 28639 & -0.026 \\
\hline 4536.043 & $\mathrm{Ti} \mathrm{I}$ & 6557 & 28596 & -0.129 \\
\hline 4548.765 & $\mathrm{Ti} \mathrm{I}$ & 6661 & 28639 & -0.274 \\
\hline 4552.456 & $\mathrm{Ti} \mathrm{I}$ & 6743 & 28703 & -0.262 \\
\hline 4656.468 & $\mathrm{Ti} \mathrm{I}$ & 0 & 21469 & -1.344 \\
\hline 4681.908 & $\mathrm{Ti} \mathrm{I}$ & 387 & 21740 & -1.129 \\
\hline
\end{tabular}


Table A.3. continued.

\begin{tabular}{|c|c|c|c|c|}
\hline$\lambda_{\text {line }}(\AA)$ & Ion & $E_{\text {low }}\left(\mathrm{cm}^{-1}\right)$ & $E_{\text {up }}\left(\mathrm{cm}^{-1}\right)$ & $\log g f$ \\
\hline 4981.732 & Ti I & 6843 & 26911 & 0.586 \\
\hline 6258.713 & Ti I & 11777 & 27750 & -0.090 \\
\hline 8382.530 & Ti I & 6599 & 18525 & -1.632 \\
\hline 8426.506 & Ti I & 6661 & 18525 & -1.253 \\
\hline 4025.140 & Ti II & 4898 & 29734 & -1.960 \\
\hline 4394.060 & Ti II & 9851 & 32603 & -1.669 \\
\hline 4395.040 & Ti II & 8744 & 31491 & -0.660 \\
\hline 4417.720 & Ti II & 9396 & 32026 & -1.240 \\
\hline 4468.500 & Ti II & 9118 & 31491 & -0.270 \\
\hline 4549.617 & Ti II & 12775 & 34748 & -0.110 \\
\hline 4563.761 & Ti II & 9851 & 31757 & -0.795 \\
\hline 4352.870 & $\mathrm{~V}_{\mathrm{I}}$ & 553 & 23520 & -0.800 \\
\hline 4379.240 & V I & 2425 & 25254 & 0.600 \\
\hline 4384.706 & V I & 553 & 23353 & -1.905 \\
\hline 4384.720 & V I & 2311 & 25112 & 0.000 \\
\hline 4395.230 & V I & 2153 & 24899 & 0.320 \\
\hline 4406.072 & V I & 8579 & 31268 & -1.000 \\
\hline 4406.640 & V I & 2425 & 25112 & -0.280 \\
\hline 4407.637 & V I & 2311 & 24993 & -0.840 \\
\hline 4408.200 & V I & 2220 & 24899 & -0.100 \\
\hline 4408.508 & V I & 2112 & 24789 & -0.610 \\
\hline 4408.512 & V I & 2153 & 24830 & -0.130 \\
\hline 4408.520 & V I & 2112 & 24789 & -0.820 \\
\hline 4419.940 & V I & 2220 & 24839 & -1.480 \\
\hline 4420.120 & V I & 2153 & 24771 & -2.252 \\
\hline 4459.760 & V I & 2311 & 24728 & -0.570 \\
\hline 4460.290 & V I & 2425 & 24839 & -0.240 \\
\hline 5698.520 & V I & 8579 & 26122 & -0.036 \\
\hline 5727.048 & V I & 8716 & 26172 & 0.088 \\
\hline 5737.059 & V I & 8579 & 26004 & -0.675 \\
\hline 6135.361 & V I & 8476 & 24771 & -0.750 \\
\hline 6150.157 & V I & 2425 & 18680 & -1.277 \\
\hline 6243.105 & V I & 2425 & 18438 & -0.878 \\
\hline 6274.649 & V I & 2153 & 18086 & -1.657 \\
\hline 6531.440 & V I & 9825 & 25131 & -1.320 \\
\hline 6531.466 & V I & 23935 & 39241 & -2.931 \\
\hline 4274.800 & Cr I & 0 & 23386 & -0.231 \\
\hline 4274.891 & Cr I & 24200 & 47586 & -2.233 \\
\hline 4289.720 & $\mathrm{Cr} \mathrm{I}$ & 0 & 23305 & -0.360 \\
\hline 4527.332 & Cr I & 20524 & 42606 & -0.906 \\
\hline 4535.695 & Cr I & 20524 & 42565 & -0.570 \\
\hline 4600.741 & Cr I & 8095 & 29825 & -1.305 \\
\hline 4600.775 & $\mathrm{Cr} \mathrm{I}$ & 23934 & 45663 & -2.354 \\
\hline 4652.152 & $\mathrm{CrI}$ & 8095 & 29585 & -1.026 \\
\hline 4708.018 & $\mathrm{Cr} I$ & 25549 & 46783 & 0.110 \\
\hline 4718.426 & Cr I & 25771 & 46959 & 0.097 \\
\hline 4829.314 & Cr I & 20524 & 41225 & -1.630 \\
\hline 4829.372 & Cr I & 20524 & 41225 & -0.787 \\
\hline 5783.886 & Cr I & 26796 & 44081 & -0.177 \\
\hline 5787.965 & $\mathrm{Cr} \mathrm{I}$ & 26796 & 44069 & 0.033 \\
\hline 5791.006 & $\mathrm{Cr} \mathrm{I}$ & 26788 & 44051 & 0.324 \\
\hline 6924.179 & Cr I & 27820 & 42258 & -0.135 \\
\hline 7462.378 & Cr I & 23499 & 36896 & -0.040 \\
\hline 8947.180 & $\mathrm{Cr} I$ & 25039 & 36212 & -0.724 \\
\hline 4554.988 & Cr II & 32837 & 54784 & -1.249 \\
\hline 4235.295 & Mn I & 23297 & 46901 & -0.030 \\
\hline 4458.260 & Mn I & 24788 & 47212 & -0.042 \\
\hline 4761.512 & Mn I & 23819 & 44815 & -0.138 \\
\hline 4762.367 & Mn I & 23297 & 44289 & 0.426 \\
\hline
\end{tabular}


A\&A 624, A1 (2019)

Table A.3. continued.

\begin{tabular}{|c|c|c|c|c|}
\hline$\lambda_{\text {line }}(\AA)$ & Ion & $E_{\text {low }}\left(\mathrm{cm}^{-1}\right)$ & $E_{\text {up }}\left(\mathrm{cm}^{-1}\right)$ & $\log g f$ \\
\hline 4765.846 & Mn I & 23720 & 44696 & -0.077 \\
\hline 4766.418 & Mn I & 23549 & 44523 & 0.098 \\
\hline 4823.524 & Mn I & 18705 & 39431 & 0.144 \\
\hline 6013.513 & Mn I & 24779 & 41404 & -0.397 \\
\hline 4045.820 & Fe I & 11976 & 36686 & 0.280 \\
\hline 4063.605 & $\mathrm{Fe}_{\mathrm{I}}$ & 12561 & 37163 & 0.072 \\
\hline 4063.627 & $\mathrm{Fe} I$ & 33096 & 57698 & -0.691 \\
\hline 4071.740 & $\mathrm{Fe} I$ & 12969 & 37521 & -0.022 \\
\hline 4271.760 & $\mathrm{Fe} \mathrm{I}$ & 11976 & 35379 & -0.164 \\
\hline 4325.739 & $\mathrm{Fe} I$ & 0 & 23111 & -4.815 \\
\hline 4325.760 & $\mathrm{Fe} \mathrm{I}$ & 12969 & 36079 & -0.300 \\
\hline 4383.550 & Fe I & 11976 & 34782 & 0.200 \\
\hline 4404.761 & $\mathrm{Fe} \mathrm{I}$ & 12561 & 35257 & -0.142 \\
\hline 5701.545 & $\mathrm{Fe}_{\mathrm{I}}$ & 20641 & 38175 & -1.565 \\
\hline 4233.113 & Fe II & 54871 & 78487 & -3.448 \\
\hline 4233.137 & Fe II & 74498 & 98115 & -2.864 \\
\hline 4233.170 & Fe II & 20831 & 44447 & -1.995 \\
\hline 4555.893 & Fe II & 22810 & 44754 & -2.281 \\
\hline 4813.449 & Co I & 23153 & 43922 & -2.121 \\
\hline 4813.467 & Co I & 25938 & 46707 & 0.050 \\
\hline 6450.247 & Co I & 13796 & 29295 & -1.698 \\
\hline 6814.942 & Co I & 15774 & 30444 & -1.700 \\
\hline 7052.868 & Co I & 15774 & 29949 & -1.440 \\
\hline 4519.979 & $\mathrm{Ni} I$ & 13521 & 35639 & -2.570 \\
\hline 4715.757 & $\mathrm{Ni} I$ & 28578 & 49778 & -0.331 \\
\hline 4786.531 & $\mathrm{Ni}$ I & 27580 & 48467 & -0.244 \\
\hline 4831.169 & $\mathrm{Ni} I$ & 29084 & 49778 & -0.291 \\
\hline 4918.362 & $\mathrm{Ni} I$ & 30980 & 51306 & -0.109 \\
\hline 4984.112 & $\mathrm{Ni} I$ & 30619 & 50678 & 0.226 \\
\hline 5892.868 & $\mathrm{Ni} I$ & 16017 & 32982 & -2.141 \\
\hline 6314.653 & $\mathrm{Ni}$ I & 15610 & 31442 & -2.402 \\
\hline 6482.810 & $\mathrm{Ni} I$ & 15610 & 31031 & -2.630 \\
\hline 6914.559 & Ni I & 15734 & 30192 & -2.270 \\
\hline 7409.346 & $\mathrm{Ni} I$ & 30619 & 44112 & -0.237 \\
\hline 7414.500 & $\mathrm{Ni}$ I & 16017 & 29501 & -2.570 \\
\hline 5700.237 & $\mathrm{Cu} \mathrm{I}$ & 13245 & 30784 & -2.312 \\
\hline 5782.127 & $\mathrm{Cu} \mathrm{I}$ & 13245 & 30535 & -1.720 \\
\hline 4810.528 & Zn I & 32890 & 53672 & -0.137 \\
\hline 7800.259 & $\mathrm{Rb} \mathrm{I}$ & 0 & 12817 & 0.137 \\
\hline 7947.597 & $\mathrm{Rb} \mathrm{I}$ & 0 & 12579 & -0.167 \\
\hline 4741.918 & Sr I & 14318 & 35400 & -0.320 \\
\hline 4872.488 & Sr I & 14504 & 35022 & -0.200 \\
\hline 6504.000 & Sr I & 18067 & 33442 & 0.260 \\
\hline 7070.070 & Sr I & 14899 & 29039 & -0.180 \\
\hline 4235.934 & Y I & 530 & 24131 & -0.490 \\
\hline 4839.855 & Y I & 11532 & 32188 & 0.480 \\
\hline 6191.718 & Y I & 0 & 16146 & -0.970 \\
\hline 6222.578 & Y I & 0 & 16066 & -1.700 \\
\hline 6435.004 & Y I & 530 & 16066 & -0.820 \\
\hline 4235.730 & Y II & 1045 & 24647 & -1.425 \\
\hline 4982.129 & Y II & 8328 & 28394 & -1.290 \\
\hline 7881.881 & Y II & 14833 & 27517 & -0.570 \\
\hline 4236.550 & $\mathrm{Zr} \mathrm{I}$ & 0 & 23604 & -1.000 \\
\hline 4772.323 & Zr I & 5023 & 25972 & 0.040 \\
\hline 4784.913 & Zr I & 5540 & 26434 & -0.490 \\
\hline 5879.782 & $\mathrm{Zr} \mathrm{I}$ & 1241 & 18244 & -1.670 \\
\hline 6127.475 & Zr I & 1241 & 17556 & -1.060 \\
\hline 6134.585 & $\mathrm{Zr} \mathrm{I}$ & 0 & 16296 & -1.280 \\
\hline
\end{tabular}


Table A.3. continued.

\begin{tabular}{|c|c|c|c|c|}
\hline$\lambda_{\text {line }}(\AA)$ & Ion & $E_{\text {low }}\left(\mathrm{cm}^{-1}\right)$ & $E_{\text {up }}\left(\mathrm{cm}^{-1}\right)$ & $\log g f$ \\
\hline 6143.252 & Zr I & 570 & 16844 & -1.100 \\
\hline 6990.869 & Zr I & 5023 & 19324 & -1.220 \\
\hline 7102.954 & $\mathrm{Zr} \mathrm{I}$ & 5249 & 19324 & -0.840 \\
\hline 8070.115 & Zr I & 5889 & 18277 & -0.790 \\
\hline 4443.000 & Zr II & 11984 & 34485 & -0.160 \\
\hline 4205.303 & $\mathrm{Nb} \mathrm{I}$ & 392 & 24165 & -0.850 \\
\hline 4523.397 & $\mathrm{Nb} \mathrm{I}$ & 1143 & 23244 & -0.800 \\
\hline 4546.818 & $\mathrm{Nb} \mathrm{I}$ & 1587 & 23574 & -0.750 \\
\hline 4573.075 & $\mathrm{Nb} I$ & 2154 & 24015 & -0.560 \\
\hline 4663.818 & $\mathrm{Nb} \mathrm{I}$ & 1587 & 23023 & -0.740 \\
\hline 5751.408 & Mo I & 11454 & 28837 & -1.014 \\
\hline 5791.839 & Mo I & 11454 & 28715 & -1.046 \\
\hline 5858.266 & Mo I & 11858 & 28924 & -0.996 \\
\hline 6619.134 & Mo I & 10768 & 25872 & -1.252 \\
\hline 4031.626 & Tc I & 2573 & 27370 & 0.39 \\
\hline 4095.668 & $\mathrm{Tc} \mathrm{I}$ & 3251 & 27660 & -0.01 \\
\hline 4238.191 & $\mathrm{Tc} \mathrm{I}$ & 0 & 23588 & -0.39 \\
\hline 4262.270 & Tc I & 0 & 23455 & -0.18 \\
\hline 4297.058 & Tc I & 0 & 23265 & -0.03 \\
\hline 4206.015 & Ru I & 8084 & 31853 & -0.480 \\
\hline 4385.385 & Ru I & 7483 & 30280 & -0.610 \\
\hline 4385.645 & Ru I & 9058 & 31853 & -0.490 \\
\hline 4410.025 & Ru I & 9184 & 31853 & -0.380 \\
\hline 4460.027 & Ru I & 8771 & 31186 & -0.530 \\
\hline 4554.517 & $\mathrm{Ru} \mathrm{I}$ & 6545 & 28495 & 0.130 \\
\hline 4554.029 & Ba II & 0 & 21952 & 0.170 \\
\hline 4934.076 & Ba II & 0 & 20262 & -0.150 \\
\hline 5853.668 & Ba II & 4874 & 21952 & -1.000 \\
\hline 6141.713 & Ba II & 5675 & 21952 & -0.076 \\
\hline 6496.930 & $\mathrm{Ba}$ II & 4874 & 20262 & 0.130 \\
\hline 4354.400 & La II & 7340 & 30305 & -0.210 \\
\hline 4354.412 & La II & 7395 & 30353 & -0.500 \\
\hline 4526.111 & La II & 6227 & 28315 & -0.770 \\
\hline 4574.860 & La II & 1394 & 23247 & -1.140 \\
\hline 4662.498 & La II & 0 & 21442 & -1.240 \\
\hline 4970.386 & La II & 2592 & 22705 & -1.190 \\
\hline 5797.565 & La II & 1971 & 19214 & -1.410 \\
\hline 5805.773 & La II & 1016 & 18236 & -1.610 \\
\hline 6390.477 & La II & 2592 & 18236 & -1.450 \\
\hline 4324.785 & Ce II & 7713 & 30829 & -0.514 \\
\hline 4324.790 & Ce II & 7662 & 30785 & -0.050 \\
\hline 4386.827 & Ce II & 1874 & 24663 & -0.582 \\
\hline 4408.851 & Ce II & 7234 & 29909 & -0.965 \\
\hline 4408.870 & Ce II & 7179 & 29860 & 0.120 \\
\hline 4408.894 & Ce II & 10314 & 32989 & -0.857 \\
\hline 4418.780 & Ce II & 6968 & 29592 & 0.310 \\
\hline 4427.916 & Ce II & 4323 & 26900 & -0.460 \\
\hline 4427.920 & Ce II & 4275 & 26859 & -0.610 \\
\hline 4427.916 & Ce II & 4323 & 26900 & -0.460 \\
\hline 4427.920 & Ce II & 4275 & 26859 & -0.610 \\
\hline 4428.438 & Ce II & 4266 & 26841 & -0.657 \\
\hline 4444.700 & Ce II & 8532 & 31024 & 0.110 \\
\hline 4483.893 & Ce II & 6968 & 29263 & 0.010 \\
\hline 4483.900 & Ce II & 6937 & 29239 & -0.050 \\
\hline 4572.278 & Ce II & 5514 & 27378 & 0.001 \\
\hline 4429.254 & Pr II & 2998 & 25569 & -0.010 \\
\hline 4205.600 & Nd II & 5086 & 28857 & 0.070 \\
\hline 4232.380 & $\mathrm{Nd}$ II & 513 & 24134 & -1.020 \\
\hline 4351.290 & Nd II & 1470 & 24445 & -1.210 \\
\hline
\end{tabular}


Table A.3. continued.

\begin{tabular}{llrrr}
\hline \hline$\lambda_{\text {line }}(\AA)$ & Ion & $E_{\text {low }}\left(\mathrm{cm}^{-1}\right)$ & $E_{\text {up }}\left(\mathrm{cm}^{-1}\right)$ & $\log g f$ \\
\hline 4358.161 & Nd II & 2585 & 25524 & -0.280 \\
4358.170 & Nd II & 4512 & 27449 & -0.060 \\
4358.161 & Nd II & 2585 & 25524 & -0.280 \\
4358.170 & Nd II & 4512 & 27449 & -0.060 \\
4385.660 & Nd II & 1650 & 24445 & -0.550 \\
4391.100 & Nd II & 2585 & 25352 & -0.240 \\
4414.440 & Nd II & 513 & 23160 & -0.840 \\
4446.390 & Nd II & 1650 & 24134 & -0.500 \\
4680.737 & Nd II & 513 & 21872 & -1.260 \\
4706.543 & Nd II & 0 & 21241 & -0.880 \\
4715.586 & Nd II & 1650 & 22850 & -1.070 \\
4820.339 & Nd II & 1650 & 22390 & -1.240 \\
4229.713 & Sm II & 327 & 23962 & -1.224 \\
4390.855 & Sm II & 1489 & 24257 & -0.804 \\
4420.524 & Sm II & 2689 & 25304 & -0.695 \\
4433.890 & Sm II & 3499 & 26046 & -0.572 \\
4676.902 & Sm II & 327 & 21702 & -1.407 \\
4522.581 & Eu II & 1669 & 23774 & -0.678 \\
6645.064 & Eu II & 11128 & 26173 & 0.204 \\
7426.569 & Eu II & 10313 & 23774 & -0.149 \\
4053.640 & Gd I & 999 & 25661 & 0.297 \\
4191.075 & Gd II & 3444 & 27298 & -0.653 \\
4394.720 & Gd II & 6533 & 29288 & -0.060 \\
4394.720 & Gd II & 6605 & 29353 & -1.783 \\
4752.526 & Tb II & 0 & 21036 & -0.816 \\
4186.819 & Dy I & 0 & 23878 & 0.693 \\
4077.966 & Dy II & 828 & 25343 & -0.058 \\
4409.383 & Dy II & 0 & 22673 & -1.420 \\
4301.596 & Er II & 0 & 23241 & -1.487 \\
4419.608 & Er II & 13572 & 36192 & 0.386 \\
7131.816 & Hf I & 0 & 14018 & -1.690 \\
7237.112 & Hf I & 4568 & 18382 & -0.840 \\
4294.605 & W I & 2951 & 26230 & -0.735 \\
4659.853 & W I & 0 & 21454 & -1.900 \\
4260.848 & Os I & 0 & 23463 & -1.440 \\
4420.468 & Os I & 0 & 22616 & -1.530 \\
4793.993 & Os I & 4159 & 25013 & -1.990 \\
\hline & & & & \\
\hline
\end{tabular}

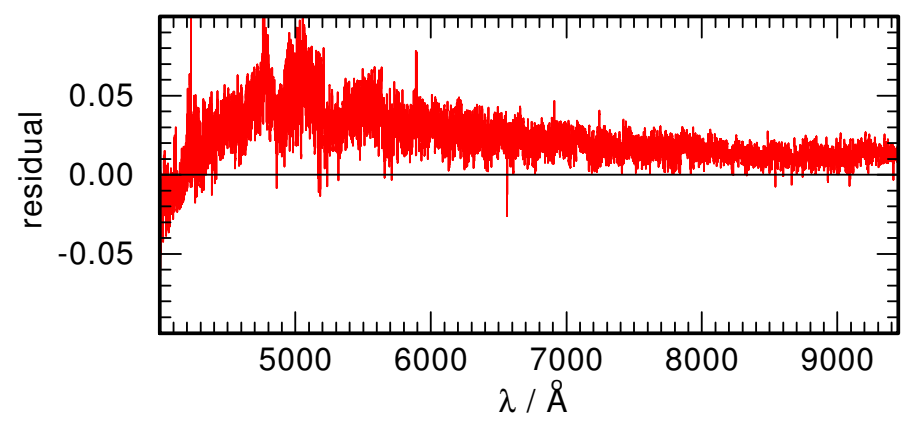

Fig. A.3. Difference between the newly computed C-rich test model (model 2) and the O-rich model from the available grid (model 1) for $T_{\text {eff }}=4250 \mathrm{~K}$ and $\log g=2.5$. 


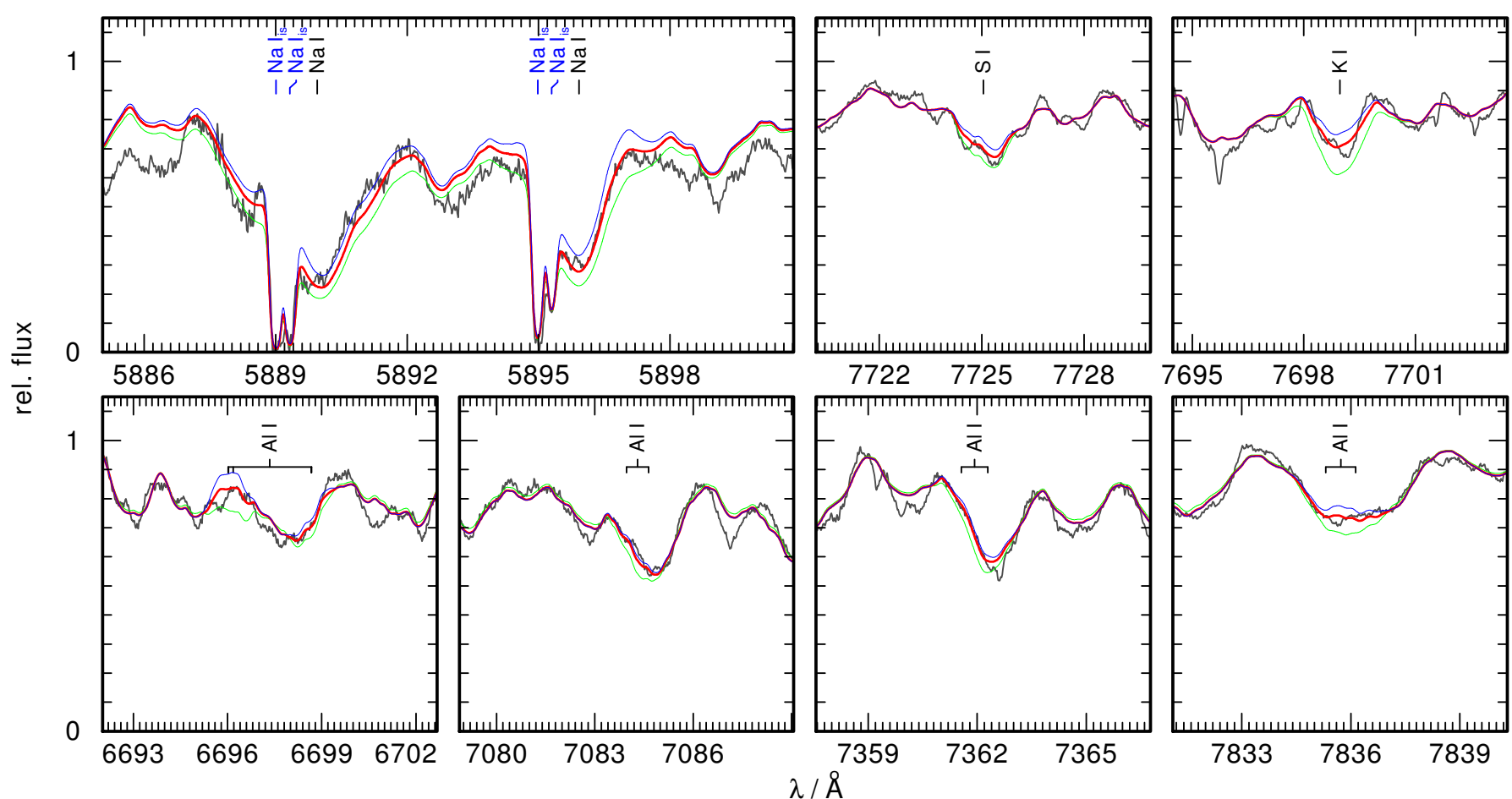

Fig. A.4. Observation (gray) of Hen 2-39 compared to model spectra for selected regions around absorption lines of NaI for $[\mathrm{Na} / \mathrm{Fe}]=0.35,0.05,-0.25$ (green, red, and blue, respectively), $\mathrm{S}$ I for $[\mathrm{S} / \mathrm{Fe}]=2.17,1.17,0.17, \mathrm{Al} \mathrm{I}$ for $[\mathrm{Al} / \mathrm{Fe}]=0.68,-0.32,-1.32$, and $\mathrm{K} \mathrm{I}$ for $[\mathrm{K} / \mathrm{Fe}]=1.07,0.07,-0.93$. Interstellar absorption lines are indicated with blue marks. 


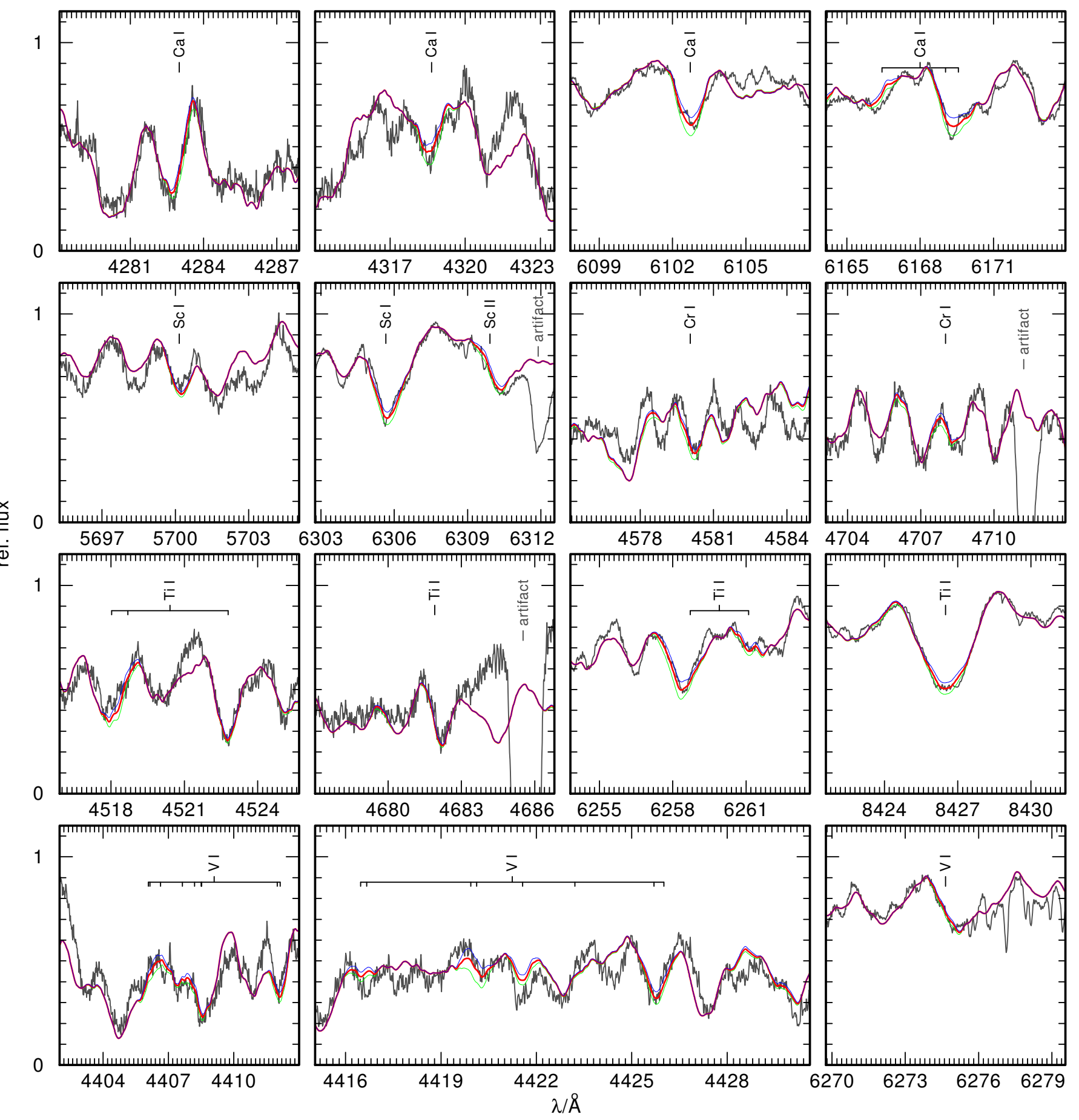

Fig. A.5. Observation (gray) of Hen 2-39 compared to model spectra for selected regions around absorption lines of $\mathrm{Ca} \mathrm{I}$ for $[\mathrm{Ca} / \mathrm{Fe}]=$ $0.41,-0.09,-0.59$ (green, red, and blue, respectively), $\mathrm{Sc}$ I for $[\mathrm{Sc} / \mathrm{Fe}]=0.08,-0.42,-0.92$, $\mathrm{Cr}$ I for $[\mathrm{Cr} / \mathrm{Fe}]=0.32,-0.18,-0.68, \mathrm{Ti}$ I for $[\mathrm{Ti} / \mathrm{Fe}]=-0.13,-0.63,-1.13$, and $\mathrm{V}$ I for $[\mathrm{V} / \mathrm{Fe}]=-0.05,-0.55,-1.05$. Artifacts arising from the overcorrection of nebula lines are indicated. 


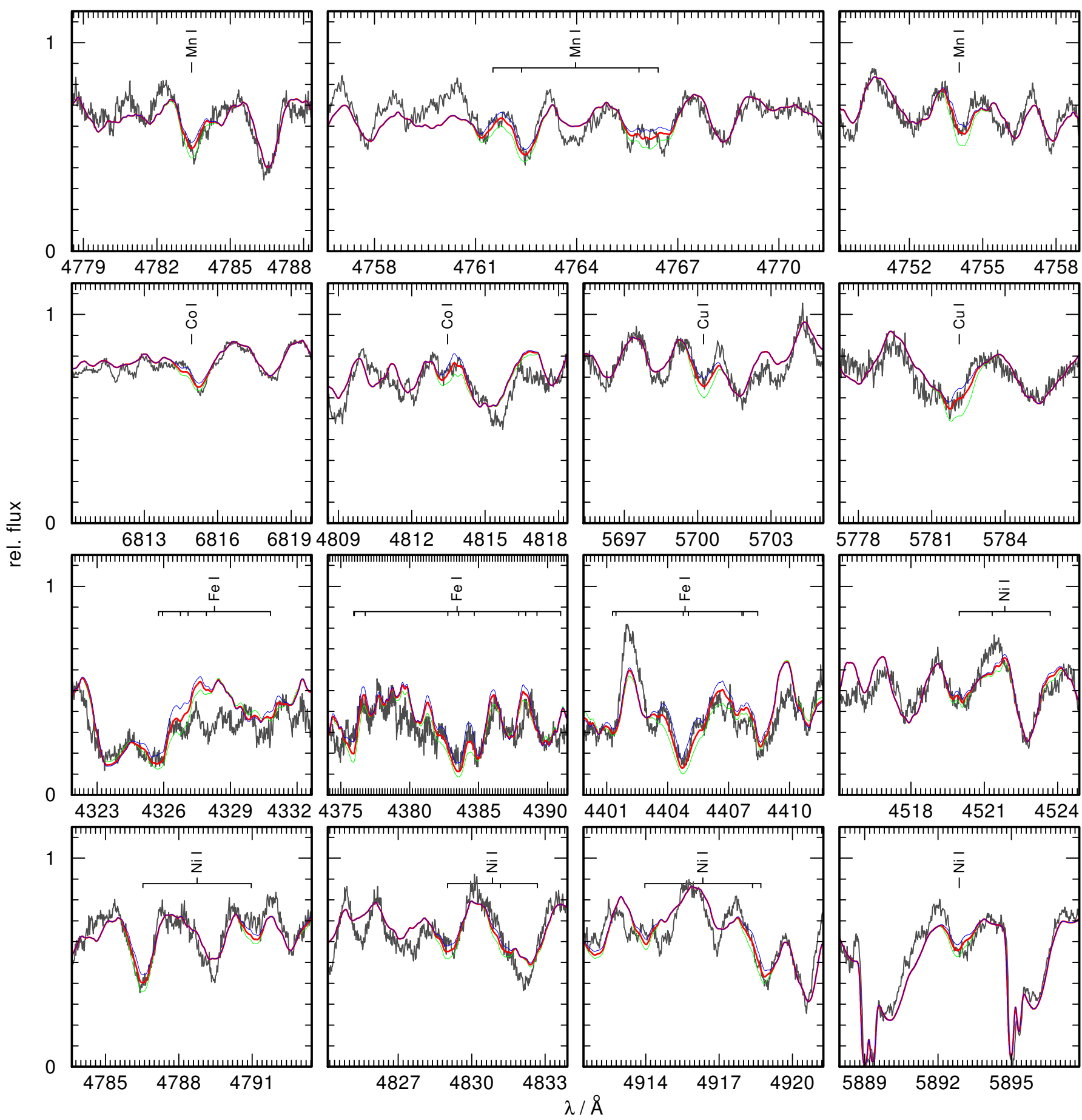

Fig. A.6. Observation (gray) of Hen 2-39 compared to model spectra for selected regions around absorption lines of MnI for $[\mathrm{Mn} / \mathrm{Fe}]=0.52,0.02,-0.48$ (green, red, and blue, respectively), Co I for $[\mathrm{Co} / \mathrm{Fe}]=1.03,0.53,0.03, \mathrm{Cu} \mathrm{I}$ for $[\mathrm{Cu} / \mathrm{Fe}]=2.18,1.18,0.18, \mathrm{Fe} \mathrm{I}$ for $[\mathrm{Fe} / \mathrm{H}]=0.15,-0.35,-0.85$, and $\mathrm{Ni}$ I for $[\mathrm{Ni} / \mathrm{Fe}]=1.09,0.59,0.09$. Interstellar absorption lines are indicated with blue marks. 

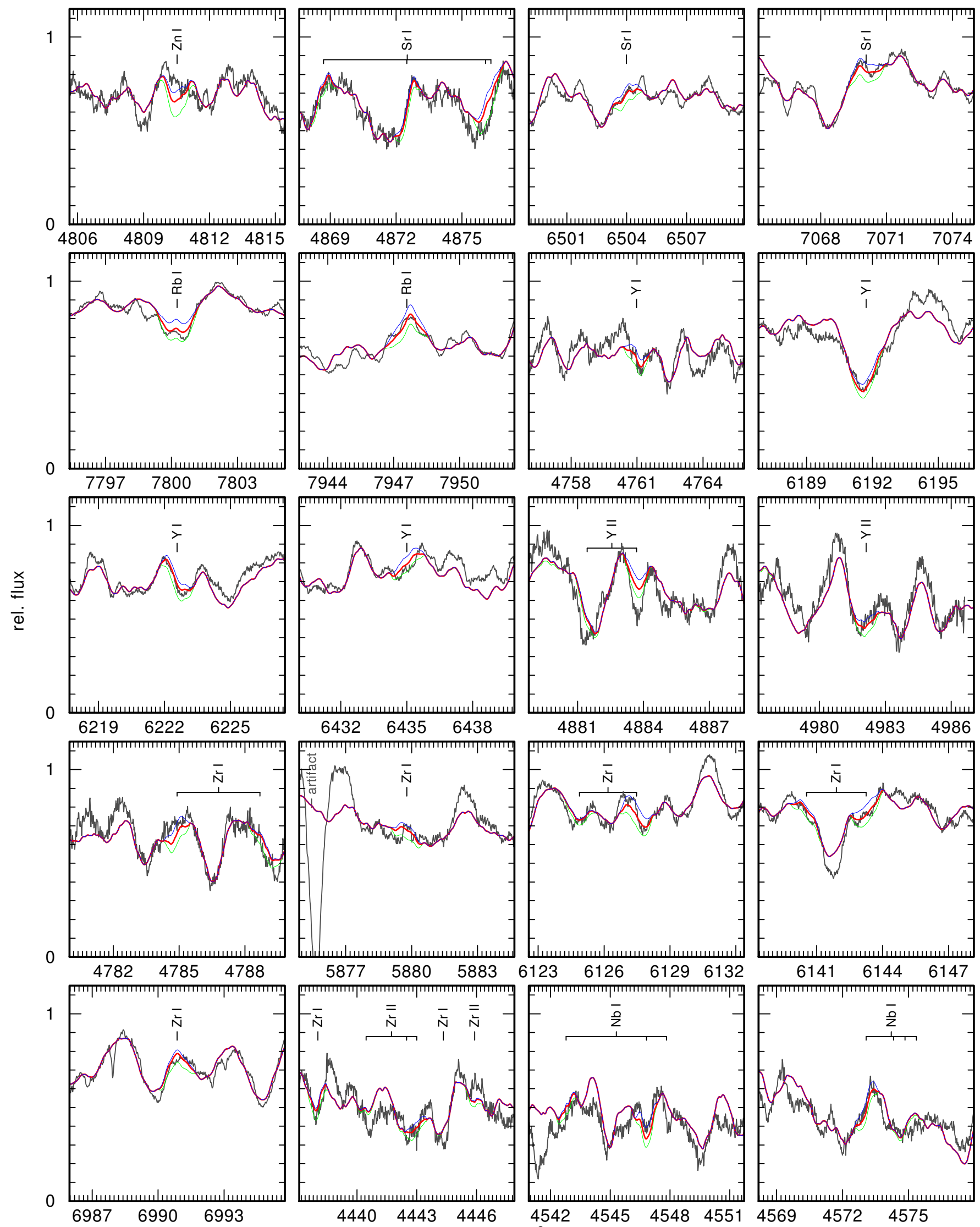

$$
\lambda / \AA
$$

Fig. A.7. Observation (gray) of Hen 2-39 compared to model spectra for selected regions around absorption lines of $\mathrm{ZnI}$ for $[\mathrm{Zn} / \mathrm{Fe}]=$ $2.51,1.51,0.51$ (green, red, and blue, respectively), $\mathrm{Sr} I$ for $[\mathrm{Sr} / \mathrm{Fe}]=1.99,0.99,-0.01, \mathrm{Rb}$ I for $[\mathrm{Rb} / \mathrm{Fe}]=2.41,1.41,0.41, \mathrm{Y} \mathrm{I}$ and $\mathrm{Y}$ II for $[\mathrm{Y} / \mathrm{Fe}]=1.37,0.37,-0.63, \mathrm{Zr}$ I and $\mathrm{Zr}$ II for $[\mathrm{Zr} / \mathrm{Fe}]=1.19,0.19-0.81$, and $\mathrm{Nb}$ I for $[\mathrm{Nb} / \mathrm{Fe}]=1.97,0.97,-0.03$. Artifacts arising from the overcorrection of nebula lines are indicated. 

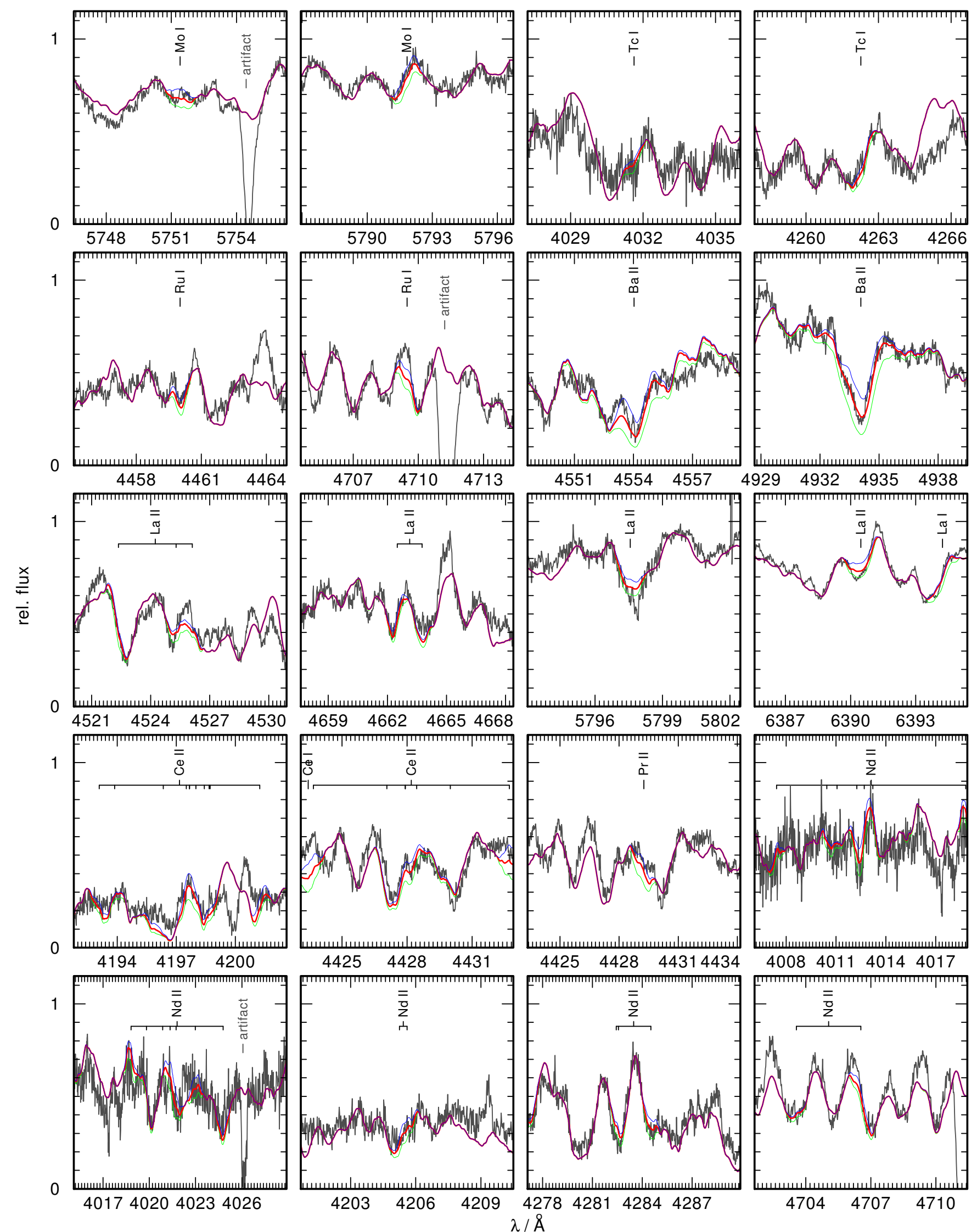

Fig. A.8. Observation (gray) of Hen 2-39 compared to model spectra for selected regions around absorption lines of Mo I for $[\mathrm{Mo} / \mathrm{Fe}]=2.35,1.35,0.35$ (green, red, and blue, respectively), $\mathrm{Tc} \mathrm{I}$ for $\log \epsilon_{\mathrm{Tc}}=3.5,2.5,1.5, \mathrm{Ru} \mathrm{I}$ for $[\mathrm{Ru} / \mathrm{Fe}]=3.05,2.05,1.05, \mathrm{Ba} \mathrm{II}$ $[\mathrm{Ba} / \mathrm{Fe}]=2.29,1.79,1.27$, La II for $[\mathrm{La} / \mathrm{Fe}]=2.44,1.44,0.44$, Ce I and Ce II for $[\mathrm{Ce} / \mathrm{Fe}]=3.29,2.29,1.29, \mathrm{Pr}$ II for $[\mathrm{Pr} / \mathrm{Fe}]=3.68,2.68,1.68$, and $\mathrm{Nd}$ I for $[\mathrm{Nd} / \mathrm{Fe}]=1.76,0.76,-0.24$. Artifacts arising from the overcorrection of nebula lines are indicated. 


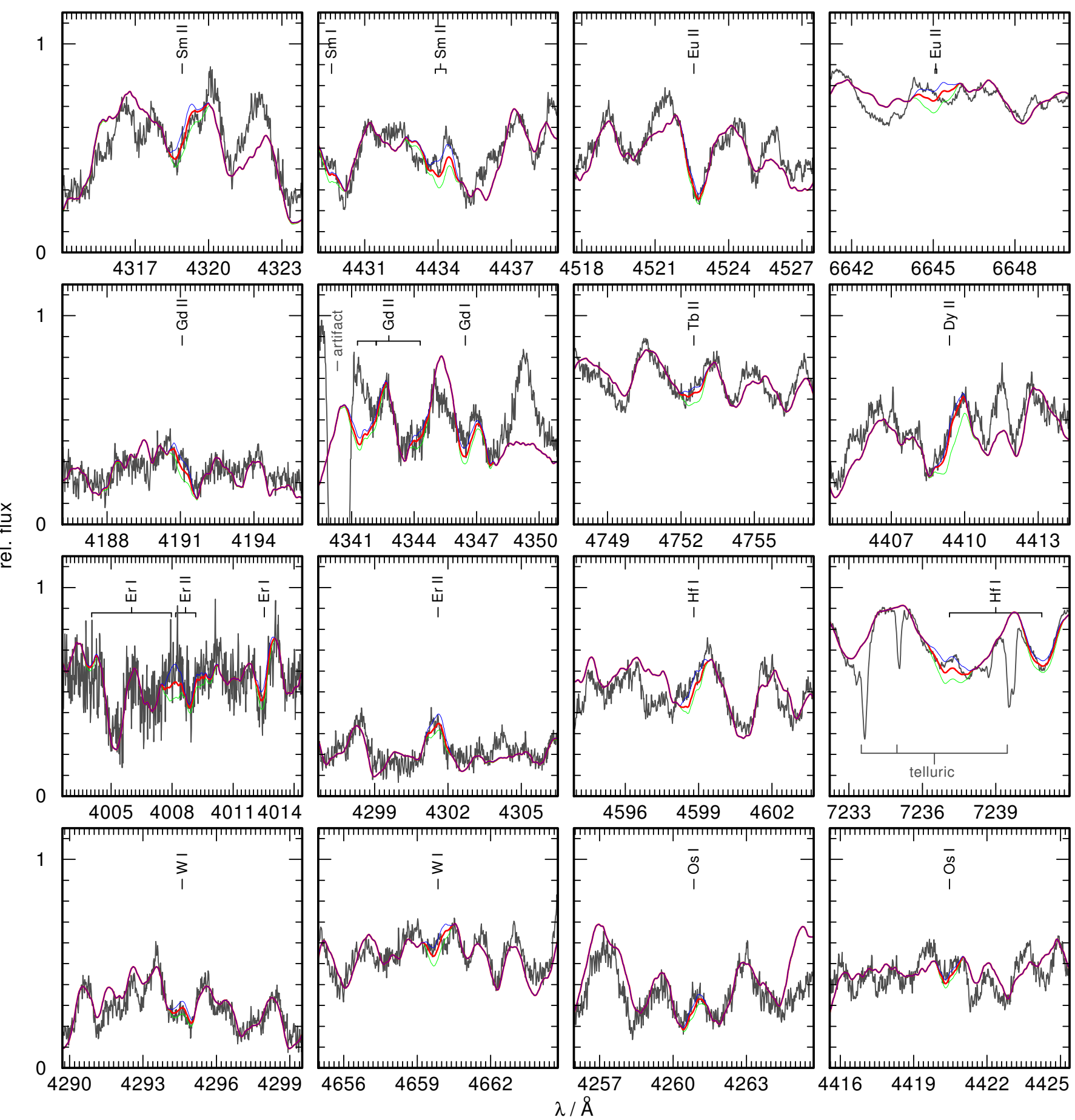

Fig. A.9. Observation (gray) of Hen 2-39 compared to model spectra for selected regions around absorption lines of Sm I and Sm II for $[\mathrm{Sm} / \mathrm{Fe}]=2.08,1.08,0.08$ (green, red, and blue, respectively), Eu II for $[\mathrm{Eu} / \mathrm{Fe}]=1.95,0.95,-0.05, \mathrm{Gd} \mathrm{I}$ and $\mathrm{Gd} \mathrm{II}[\mathrm{Gd} / \mathrm{Fe}]=2.80,1.80,0.80$, Tb II for $[\mathrm{Tb} / \mathrm{Fe}]=1.79,0.79,-0.21$, Dy II for $[\mathrm{Dy} / \mathrm{Fe}]=4.75,3.75,2.75$, ErI and $\mathrm{Er}$ II $[\mathrm{Er} / \mathrm{Fe}]=2.86,1.86,0.86$, Hf I for $[\mathrm{Hf} / \mathrm{Fe}]=$ $2.31,1.31,0.31$, W I for $[\mathrm{W} / \mathrm{Fe}]=2.05,1.05,0.05$, and Os I for $[\mathrm{Os} / \mathrm{Fe}]=2.76,1.76,0.76$. Artifacts arising from the overcorrection of nebula lines are indicated. 